\title{
Diffusion of spheres in isotropic and nematic suspensions of rods
}

\author{
Kyongok Kang \\ Forschungszentrum Jülich, Institute für Festkörper Forschung (IFF), Weiche Materie, D-52425 Julich, \\ Germany
}

A. Wilk

Institute of Physics, A. Mickiewicz University, Umultowska 85, 61-614 Poznan, Poland

J. Buitenhuis

Forschungszentrum Jülich, Institute für Festkörper Forschung (IFF), Weiche Materie, D-52425 Julich, Germany

\author{
A. Patkowski \\ Institute of Physics, A. Mickiewicz University, Umultowska 85, 61-614 Poznan, Poland \\ Jan K. G. Dhont ${ }^{a)}$ \\ Forschungszentrum Jülich, Institute für Festkörper Forschung (IFF), Weiche Materie, D-52425 Julich, \\ Germany
}

(Received 6 September 2005; accepted 28 November 2005; published online 26 January 2006)

\begin{abstract}
Diffusion of a small tracer sphere (apoferritin) in isotropic and nematic networks [of fd virus] is discussed. For a tracer sphere that is smaller than the mesh size of the network, screened hydrodynamic interactions between the sphere and the network determine its diffusion coefficient. A theory is developed for such interactions as well as their relation to the long-time self-diffusion coefficient. Fluorescence correlation spectroscopy measurements on mixtures of apoferritin and fd virus are presented. The long-time self-diffusion coefficient of apoferritin is measured as a function of the fd-virus concentration, both in the isotropic and nematic state, in directions parallel and perpendicular to the nematic director. The hydrodynamic screening length of the fd-virus network as a function of fd concentration is obtained by combining these experimental data with the theory. Surprisingly, the screening length increases with increasing concentration in nematic networks. This is due to the increase in the degree of alignment, which apparently leads to a strong increase of the screening length. Hydrodynamic screening is thus strongly diminished by alignment. A self-consistent calculation of the screening length does not work at higher concentrations, probably due to the strong variation of the typical incident flow fields over the contour of a rod. (C) 2006 American Institute of Physics. [DOI: 10.1063/1.2161204]
\end{abstract}

\section{INTRODUCTION}

Diffusion in various types of lyotropic mixtures have been of increasing interest, not only since this is interesting in itself but also due to its biological relevance: tracer diffusion in networks (of rods) is probably relevant for mass transport of proteins in the cell. As such, most of the investigations have been focused on diffusion of spherelike tracers in $F$-actin networks/cytoplasm and their mechanical properties, where tracer spheres are sometimes used to probe microrheological response. ${ }^{1-11}$

To gain in fundamental understanding on diffusion of spheres through networks we have performed fluorescence correlation spectroscopy (FCS) experiments on a relatively simple model system: apoferritin as a tracer particle and a (isotropic or nematic) network of feline distemper (fd)-virus particles. Apoferritin is a semispherical protein with a diameter of $12.8 \mathrm{~nm}$, while fd virus is a very long and thin rod (contour length of $880 \mathrm{~nm}$ and thickness of $6.8 \mathrm{~nm}$ ), which is relatively stiff (persistence length is $2200 \mathrm{~nm}$ ). Fd virus has

${ }^{a)}$ Electronic mail: j.k.g.dhont@fz-juelich.de been shown to be a very nice model system for rodlike colloids which exhibit an isotropic-nematic-smectic phase transition. $^{12-16}$ Some (micro-) rheological experiments have been performed on fd suspensions. ${ }^{17-20}$ Mixtures of apoferritin and fd virus are stable at high salt concentrations (experiments are done at $110 \mathrm{mM}$ ). The interactions between apoferritin and fd-virus particles and between different $\mathrm{fd}$ rods are therefore almost hard-core-like. The diameter of the tracer protein is small in comparison with the typical mesh size of the fd-virus network, and is comparable with the thickness of the rods. Spheres with a diameter substantially larger than the diameter of a rod do not dissolve in a lyotropic nematic. It is therefore natural to study diffusion of small spheres as far as nematic networks are concerned. The existing theories on diffusion through networks/porous media are either microscopic theories where diffusion through "networks" of fixed spheres/points are considered (see Refs. 21-24 and older references therein) or phenomenological, qualitative results are derived (see Refs. 25-27). In Ref. 28, the effect of a fibrous medium on the concentration dependence of the gradient-diffusion coefficient of spheres is discussed. As far as we know, there is no microscopic theory 
concerned with self-diffusion of spheres through open networks of rodlike obstacles. For the open networks considered here, (screened) hydrodynamic interactions between the sphere and the rods determine the diffusive properties to a large extent. A semiquantitative theory is developed in the present paper to describe the diffusion of a small tracer sphere in an open network of stiff rods, within a similar framework as developed in Refs. 29 and 30 for flexible polymers, where the prediction of the hydrodynamic screening length remains an open problem.

In a previous paper, ${ }^{31}$ diffusion of colloidal tracer spheres of various diameters in isotropic fd-virus suspensions, with concentrations substantially below the isotropicnematic transition concentration, has been studied. FCS, dynamic light-scattering, and video microscopy experiments were performed depending on the diameter of the tracer sphere. Furthermore, a theory is developed in that paper for tracer diffusion of large tracer spheres at low concentrations of rods. In the present paper the other limiting case of very small tracers is considered, both in isotropic and nematic rod dispersions.

Diffusion of spherical-like tracers in other types of host matrices have also been investigated, such as aqueous solutions of polystyrene sulfonate, ${ }^{32}$ dextran solution, ${ }^{33}$ flexible poly(acrylamide) gels, ${ }^{34}$ agarose gels, ${ }^{35}$ dendrites of cultured mitral cells, ${ }^{36}$ polymer solutions, ${ }^{37}$ semidilute DNA solutions, ${ }^{38}$ and in dispersions of xanthan. ${ }^{39}$

Little work has been done on tracer diffusion of rods. Van Bruggen et al. ${ }^{40,41}$ used fluorescence recovery after photobleaching (FRAP) to study self-diffusion of Boehmite rods in concentrated dispersions, where both diffusions parallel and perpendicular to the nematic director are probed. The same technique has been employed by Lellig $\mathrm{et} \mathrm{al} .{ }^{42}$ to probe diffusion of tobacco mosaic virus (TMV) in a host matrix of charged fluorinated latex spheres. Video (confocal) microscopy has been employed very recently to measure the selfdiffusion coefficient of fd virus in concentrated dispersion, again both parallel and perpendicular to the director. ${ }^{43}$ There is a large difference between the diffusion coefficients related to diffusion parallel and perpendicular to the director.

The long-time self-diffusion coefficient can be obtained from the relation, ${ }^{44}$

$$
D_{s}=k_{B} T / \gamma_{p},
$$

where $k_{B}$ is Boltzmann's constant, $T$ the temperature, and $\gamma_{p}$ the friction coefficient of the tracer particle with its surrounding, including both frictions with the solvent and interactions with other colloidal particles. The relation (1) can be derived from a Langevin equation in precisely the same way as the original Einstein relation for the diffusion coefficient of a single sphere in an otherwise unbounded fluid. The fluctuating force in the Langevin equation now includes forces due to interactions with other colloidal particles. Coarse graining to a time scale that is long in comparison with typical colloid relaxation times renders the correlation function of this fluctuating force delta correlated in time. Such a delta correlation of forces leads to the relation between the long-time selfdiffusion coefficient and the friction coefficient as given in Eq. (1). In case of tracer diffusion of a sphere through a
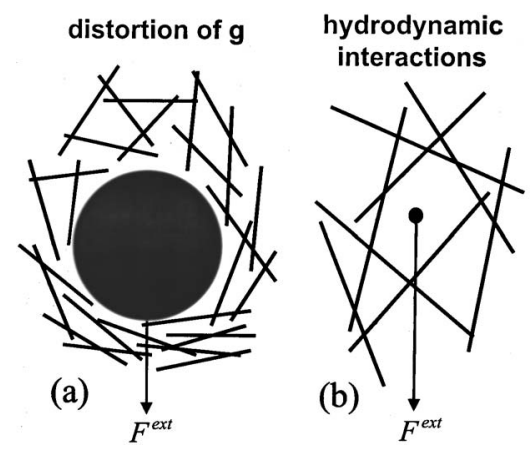

distortion of $\mathbf{g}$ ("shadowing")

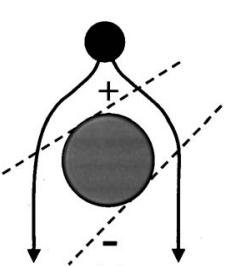

(c)

FIG. 1. (a) The effective friction coefficient for a big sphere is preliminary determined by the distortion of the pair-correlation function $g$ between the tracer sphere and the rods. Here, microstructural order of the network is severely distorted on pulling the tracer sphere through the network. (b) For a small sphere in an open network, hydrodynamic interactions with the essentially undistorted network determine the effective friction coefficient. (c) Even when the network structure is not affected by pulling the tracer sphere through the network, the pair-correlation function $g$ will be distorted by the external force on the tracer sphere. The gray circle is a cross section through the core of a rod. The + and - denote an increased and decreased pair-correlation function due to "shadowing," respectively.

network of rods, the friction coefficient is generally determined by both hydrodynamic and direct interactions of the tracer sphere with the rods. For tracer spheres which are small in comparison with the mesh size of the network, it will turn out that the hydrodynamic contribution is dominant.

The friction coefficient connects a small external force $\mathbf{F}^{\text {ext }}$ acting on the sphere with the resulting ensembleaveraged velocity $\mathbf{v}_{p}$ of the tracer sphere as

$$
\mathbf{F}^{\mathrm{ext}}=\gamma_{p} \mathbf{v}_{p} .
$$

In order to obtain the friction coefficient $\gamma$, one should thus calculate the ensemble-averaged velocity of the tracer sphere due to a small external force.

The relation (1) no longer holds in case the dynamics of the network is very slow, such that the duration of an experiment is not very much larger than the corresponding network relaxation time. In this case, the above-mentioned coarse graining to render forces delta correlated in time is not achieved. Anomalous diffusion will then be observed in the sense that the mean-squared displacement is not linear in time. Diffusion properties should now be obtained from an analysis of the Langevin equation where the forces are not delta correlated.

In the absence of such anomalous diffusion, the following two limiting cases can be distinguished when considering self-diffusion of spheres in networks of rods.

(i) The size of the tracer sphere is large compared with the mesh size of the network of the rods. In this case, microstructural order of the network of rods is severely distorted as the tracer sphere is pulled through the network [as depicted in Fig. 1(a)]. The rod concentration is enhanced in front of the moving tracer sphere, while it is decreased in its wake. In addition, orientational order of rods around the tracer sphere will be affected. The accompanied distortion of the tracer-host pair-correlation function determines the friction coefficient. Hydrodynamic interactions be- 
tween the tracer sphere and the rods are less important for this case.

(ii) The size of the tracer sphere is small compared with the mesh size of the network. In this case the distortion of microstructural order of the network as the tracer sphere is pulled through is much less important, since the sphere can simply move through the "holes" in the network. Microstructural order of the rod network can be considered unaffected by the tracer sphere. The effective friction coefficient is now determined by hydrodynamic interactions between the tracer sphere and the rods [see Fig. 1(b)] as well as the distortion of the pair-correlation function for tracer sphere and rods. Although the network structure is unaffected by pulling the small tracer sphere through the network, the tracer-host pair-correlation function will still be distorted through the "probability shadow" that a rod creates [as depicted in Fig. 1(c)]: the probability to find the sphere above the rod is higher than below, since the sphere has to avoid the core of the rod on passing it. The latter phenomenon will be referred to here as the "shadowing effect."

A variational approach has been developed in Ref. 31 for case (i) for rod concentrations below the overlap concentration. For higher concentrations, above the overlap concentration, correlations between different rods must be included. The variational approach might be generalized to take such correlations into account.

In the present paper, we will consider case (ii), where microstructural order of the network is not affected by the tracer sphere. Hydrodynamic interactions as well as the "shadowing distortion" of the pair-correlation function will be addressed in the present paper. Theoretical predictions for the hydrodynamic and shadowing contributions to the diffusion coefficient are made in Sec. II. Due to strong entanglement of the rods, hydrodynamic interactions between the tracer sphere and the rods are screened. There is no independent theory for the screening length. The screening length will be an adjustable parameter when comparing experimental results with the theory developed in Sec. II. At the end of the theoretical summary in Sec. II C we will show that the long-time self-diffusion coefficient is equal to Fick's gradient diffusion coefficient, provided that the network structure is not affected by the presence of the tracer spheres. The longtime self-diffusion coefficient is thus relevant for mass transport of dilute dispersions of small spheres in open networks. Section III introduces the colloidal particles that have been used in the experimental study. In Sec. IV tracer diffusion coefficients as obtained from FCS are presented, and the theory discussed in Sec. II is used to extract the hydrodynamic screening length from these data, both for isotropic and nematic networks of rods. Section V is a summary and discussion.

\section{THEORY}

In this section, tracer diffusion of a small sphere through an open network is discussed, corresponding to the limiting situation of case (ii) mentioned in the Introduction. In such

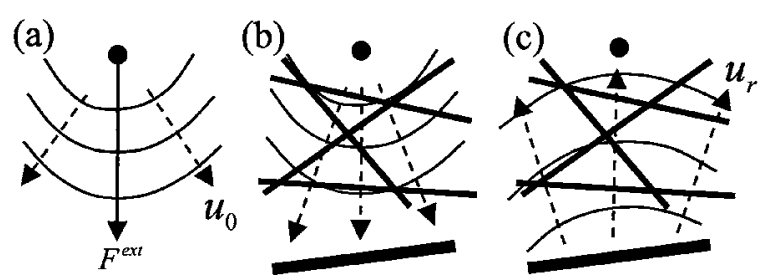

FIG. 2. (a) The flow field $\mathbf{u}_{0}$ generated by a moving sphere in an unbounded fluid. (b) The flow field $\mathbf{u}_{0}$ generated by the moving sphere traverses through the network before reaching the rod with which hydrodynamic interaction is considered. (c) The reflected flow field $\mathbf{u}_{r}$ from the rod under consideration traverses through the network back to the tracer sphere.

an open network, the structure of the network will only be slightly affected when pulling the small tracer sphere through the network. Neglecting the network distortion, hydrodynamic interaction of the tracer sphere with the rods and the shadowing effect determine the diffusion coefficient. The hydrodynamic contribution is discussed in Sec. II A and the shadowing effect is discussed in Sec. II B. As will turn out, the hydrodynamic contribution is dominant over the shadowing effect for small tracer spheres.

\section{A. The hydrodynamic contribution}

Consider the tracer sphere on which an external force $\mathbf{F}^{\text {ext }}$ acts which is such that it sustains a prescribed velocity $\mathbf{v}_{p}$ of the tracer particle. The moving tracer sphere induces a fluid flow $\mathbf{u}_{0}$ [see Fig. 2(a)]. Each of the rods in the network will reflect the incident flow field $\mathbf{u}_{0}$. The fluid flow $\mathbf{u}_{r}$ that is reflected by each rod affects the force $\mathbf{F}^{\text {ext }}$ that is necessary to sustain the prescribed velocity of the tracer sphere [see Figs. 2(b) and 2(c)]. This contributes to the effective friction coefficient of the sphere, and thus to a rod-concentrationdependent diffusion coefficient. Since the network is assumed to be relatively open to the tracer sphere, higher-order reflections of flow fields are relatively unimportant as compared with the leading order reflected field $\mathbf{u}_{r}$ by each individual rod. Second- and higher-order reflected fields will be neglected. Considering hydrodynamic interaction between the tracer sphere and a given rod, there is an influence of the rods in between the sphere and the particular rod due to "hydrodynamic screening." Above the overlap concentration of rods, each rod is assumed to be entangled to such an extent that it will be fixed in space on reflection of the incident fluid flow $\mathbf{u}_{0}$. The force on each rod due to the incident field $\mathbf{u}_{0}$ is thus assumed to be counterbalanced by its interactions with other rods in the network. The total force that the flow $\mathbf{u}_{0}$ exerts on the rod is thus nonzero, which leads to hydrodynamic screening. Both the field $\mathbf{u}_{0}$ generated by the moving sphere and the reflected flow field $\mathbf{u}_{r}$ from a given rod traverse through the network of entangled rods, as depicted in Figs. 2(b) and 2(c). The hydrodynamic influence of the rods, intermediate between the sphere and the particular rod under consideration, can be described in a mean-field approximation by adding a body force to the creeping flow equations. This body force is equal to the total force (per unit volume) that the entangled rods exert on the fluid. The force with which a single rod, immersed in a flow field $\mathbf{u}$, acts onto the fluid is equal to 


$$
\mathbf{F}=-\lambda \mathbf{u},
$$

where $\lambda$ is an "effective friction coefficient." The corresponding body force is thus equal to

$$
\mathbf{f}=-\bar{\rho} \lambda \mathbf{u},
$$

with $\bar{\rho}$ the number density of rods. This can be alternatively written as

$$
\mathbf{f}=-\eta_{0} \kappa^{2} \mathbf{u} \quad \text { with } \kappa=\sqrt{\bar{\rho} \lambda / \eta_{0}},
$$

where $\eta_{0}$ is the shear viscosity of the fluid and "the hydrodynamic screening length" $1 / \kappa$ is a measure for the distance over which screening becomes effective. Aside from body forces $\eta_{0} \nabla^{2} \mathbf{u}$ due to friction, and body forces, $\nabla p$ arising from gradients in the pressure $p$, there is now a body force (5) from the intervening rods between the tracer sphere and the particular rod under consideration. The creeping flow equation is thus replaced by the Debye-Büche-Brinkman equation,

$$
\eta_{0} \nabla^{2} \mathbf{u}-\nabla p-\eta_{0} \kappa^{2} \mathbf{u}=0, \quad \nabla \cdot \mathbf{u}=0 .
$$

The Green's function for the fluid flow velocity of this equation reads

$$
\begin{aligned}
& \mathbf{T}_{s}(\mathbf{r})=\frac{1}{4 \pi \eta_{0} r}\left[h_{1}(x) \hat{\mathbf{I}}+h_{2}(x) \hat{\mathbf{r}} \mathbf{r}\right], \\
& h_{1}(x)=-x^{-2}+\exp \{-x\}\left(1+x^{-1}+x^{-2}\right), \\
& h_{2}(x)=3 x^{-2}-\exp \{-x\}\left(1+3 x^{-1}+3 x^{-2}\right),
\end{aligned}
$$

where $\hat{\mathbf{I}}$ is the identity and $x=\kappa r$, with $\mathbf{r}$ the distance between the positions of the point where a force acts and the point where the resulting fluid flow velocity is measured, while $\hat{\mathbf{r}}$ $=\mathbf{r} / r$ is the unit vector along $\mathbf{r}$. The subscript $s$ stands for "screening." For short distances or long screening lengths,

$$
h_{1}(x)=1 / 2=h_{2}(x) \quad \text { for } x \downarrow 0,
$$

in which case the screened Oseen tensor (7) reduces to the Oseen tensor for the creeping flow equations in the absence of the body force (5). Contrary to the Oseen tensor, the screened Oseen tensor varies like $\sim r^{-3}$ for large distances. This will turn out to be important for the convergence of ensemble averages that determine the diffusion coefficient.

For a nematic network of rods, the screening length is in principle anisotropic. However, since the flow induced by a sphere is stronger in the direction of motion of the sphere, it will be assumed here that the most important contributions to hydrodynamic interactions relates to the fluid flow in the direction of motion of the tracer sphere (which is either parallel or perpendicular to the director of the nematic network). The validity of this assumption needs further theoretical work, where the Green's function of the Debye-BücheBrinkman equation with an anisotropic screening should be computed. As far as we know this has not been done yet, and is outside the scope of the present paper.

The mean-field concept of hydrodynamic screening makes sense only when the hydrodynamic screening length $1 / \kappa$ is larger than the mesh size of the network. For the open networks under consideration, the mesh size is much larger than both the thickness $D$ of the rods and the diameter $2 a$ of the tracer sphere. We shall therefore assume hereafter that $\kappa D$ and $\kappa a$ are small numbers. The induced hydrodynamic force distribution over the surface of the tracer sphere is therefore not affected by screening. Furthermore, the typical distance between the tracer sphere and rods in the relatively open network will be large in comparison with the radius of the sphere. Within these limits, the velocity field $\mathbf{u}_{0}$ of the moving tracer sphere with velocity $\mathbf{v}_{p}$ in an otherwise quiescent and unbounded, screened fluid is equal to

$$
\mathbf{u}_{0}(\mathbf{r})=6 \pi \eta_{0} a \mathbf{T}_{s}\left(\mathbf{r}-\mathbf{r}_{p}\right) \cdot \mathbf{v}_{p},
$$

with $\mathbf{r}_{p}$ the position of the center of the sphere.

Once the reflected field $\mathbf{u}_{r}$ by a rod immersed in the velocity field $\mathbf{u}_{0}$ is known, the force $\mathbf{F}^{\text {ext }}$ on the tracer sphere that is necessary to maintain its velocity $\mathbf{v}_{p}$ follows from Faxén's theorem for a Debye-Büche-Brinkman fluid. ${ }^{28}$ Since the screening length is large in comparison with the radius of the sphere, however, Faxén's theorem for an unscreened fluid can be used here, that is,

$$
\mathbf{F}^{\mathrm{ext}}=6 \pi \eta_{0} a\left[\mathbf{v}_{p}-\mathbf{u}_{r}\left(\mathbf{r}=\mathbf{r}_{p}\right)\right],
$$

where it is assumed that the variation of the reflected flow field over the sphere's surface can be neglected. This is typically the case for the small sphere in the open network of rods under consideration here.

The calculation of the field $\mathbf{u}_{r}$ that is reflected by a single rod immersed in the flow field $\mathbf{u}_{0}$ proceeds as follows.

The rod under consideration is modeled as a rigid string of $2 m+1$ spherical beads with diameter $D$ equal to the thickness of the rod. Each bead is indexed by a number $\alpha$, which ranges from $-m$ to $+m$. The value of $m$ is set by the aspect ratio as $2 m+1=L / D$, with $L$ the length of the rod. The position coordinate of the $\alpha$ th bead is written as $\mathbf{r}_{c}+D \alpha \hat{\mathbf{u}}$, where $\mathbf{r}_{c}$ is the center position of the rod (the position the bead with index number 0 ), and the unit vector $\hat{\mathbf{u}}$ defines the orientation of the long axis of the rod.

The fluid flow velocity $\mathbf{u}_{r}(\mathbf{r})$ at point $\mathbf{r}$ that is reflected by the rod after insertion in the flow field $\mathbf{u}_{0}$ generated by the moving sphere is thus written as a sum over beads which constitute the rod under consideration,

$$
\mathbf{u}_{r}(\mathbf{r})=\sum_{\alpha} \mathbf{T}_{s}\left(\mathbf{r}-\mathbf{r}_{c}-D \alpha \hat{\mathbf{u}}\right) \cdot \mathbf{F}_{\alpha}^{h},
$$

where $\mathbf{F}_{\alpha}^{h}$ is the force that bead $\alpha$ exerts on the fluid. As before, the assumption here is that the hydrodynamic screening length is large as compared with the thickness of the rod. For the open networks under consideration, the incident flow $\mathbf{u}_{0}$ varies little over distances of order $D$, so that Faxén's theorem for bead $\alpha$ reads

$$
\mathbf{F}_{\alpha}^{h}=3 \pi \eta_{0} D\left[\mathbf{v}_{\alpha}-\mathbf{u}_{\alpha}^{\star}\left(\mathbf{r}=\mathbf{r}_{c}+D \alpha \hat{\mathbf{u}}\right)\right],
$$

where $\mathbf{v}_{\alpha}$ is the velocity of bead $\alpha$, and $\mathbf{u}_{\alpha}^{\star}$ is the total flow velocity that would exist in the absence of bead $\alpha$ (as indicated by the $\star$ ). Assuming that the rod is fixed in space,

$$
\mathbf{v}_{\alpha}=0 .
$$

Furthermore, the velocity $\mathbf{u}^{\star}$ that would have existed in the absence of bead $\alpha$ is equal to 


$$
\mathbf{u}_{\alpha}^{\star}(\mathbf{r})=\mathbf{u}_{0}(\mathbf{r})+\sum_{\beta \neq \alpha} \mathbf{T}_{s}\left(\mathbf{r}-\mathbf{r}_{c}-D \beta \hat{\mathbf{u}}\right) \cdot \mathbf{F}_{\beta}^{h, \star}
$$

Here, $\mathbf{F}_{\beta}^{h, \star}$ is the force of bead $\beta$ on the fluid, in the absence of bead $\alpha$. The use of the screened Oseen tensor here accounts for screening of the flow field generated by beads $\beta$ during its propagation to bead $\alpha$, typically over distances of the order of the length $L$ of the rod. For very long and thin rods, the forces $\mathbf{F}_{\beta}^{h, \star}$ are to a good approximation equal to the forces $\mathbf{F}_{\beta}^{h}$ of the intact rod, except for the relatively small number of beads close to bead $\alpha$. This approximation leads to correct asymptotic expressions for friction coefficients of single, very long, and thin rods. Hence, we will replace $\mathbf{F}_{\beta}^{h, \star}$ by $\mathbf{F}_{\beta}^{h}$. The expressions (11)-(14) thus lead to the following "integral equation" for the hydrodynamic forces,

$$
\begin{aligned}
& \frac{\mathbf{F}_{\alpha}^{h}}{3 \pi \eta_{0} D}+\mathbf{u}_{0}\left(\mathbf{r}=\mathbf{r}_{c}+D \alpha \hat{\mathbf{u}}\right)+\sum_{\beta \neq \alpha} \mathbf{T}_{s}(D \hat{\mathbf{u}}(\alpha-\beta)) \cdot \mathbf{F}_{\beta}^{h} \\
& \quad=0 .
\end{aligned}
$$

Consider the bead-index sum in Eq. (15). Using Eq. (7) for the screened Oseen tensor, this sum can be written as

$$
\begin{aligned}
\sum_{\beta \neq \alpha} \mathbf{T}_{s}(D \hat{\mathbf{u}}(\alpha-\beta)) \cdot \mathbf{F}_{\beta}^{h} \\
=\frac{1}{4 \pi \eta_{0} D} \mathbf{F}_{\alpha}^{h} \cdot \sum_{\beta \neq \alpha} \frac{1}{|\beta-\alpha|}\left[h_{1}(\kappa D|\alpha-\beta|) \hat{\mathbf{I}}\right. \\
\left.\quad+h_{2}(\kappa D|\alpha-\beta|) \hat{\mathbf{u}} \hat{\mathbf{u}}\right] \\
\quad+\frac{1}{4 \pi \eta_{0} D} \sum_{\beta \neq \alpha} \frac{\mathbf{F}_{\beta}^{h}-\mathbf{F}_{\alpha}^{h}}{|\beta-\alpha|} \cdot\left[h_{1}(\kappa D|\alpha-\beta|) \hat{\mathbf{I}}\right. \\
\left.\quad+h_{2}(\kappa D|\alpha-\beta|) \hat{\mathbf{u}} \hat{\mathbf{u}}\right] .
\end{aligned}
$$

The first sum on the right-hand side is much larger than the second sum, since in the second sum the value of $\mid \mathbf{F}_{\beta}^{h}$ $-\mathbf{F}_{\alpha}^{h} / /|\beta-\alpha|$ is typically much smaller than $\left|\mathbf{F}_{\alpha}^{h} / /\right| \beta-\alpha \mid$. The second sum is therefore neglected. In addition, for very long and thin rods, for the majority of beads, the first sum is only slightly depending on $\alpha$. The $\alpha$ dependence in the first sum will therefore be neglected. These two approximations [neglecting the second sum in Eq. (16) and neglecting end effects in the first sum] are discussed in detail in Appendix A, where it is shown that the errors made are typically about $10 \%$. Hence, Eq. (16) is approximated as

$$
\begin{aligned}
& \sum_{\beta \neq \alpha} \mathbf{T}_{s}(D \hat{\mathbf{u}}(\alpha-\beta)) \cdot \mathbf{F}_{\beta}^{h} \\
& \quad=\frac{1}{4 \pi \eta_{0} D} \mathbf{F}_{\alpha}^{h} \cdot \sum_{\beta \neq 0} \frac{1}{|\beta|}\left[h_{1}(\kappa D|\beta|) \hat{\mathbf{I}}+h_{2}(\kappa D|\beta|) \hat{\mathbf{u}} \hat{\mathbf{u}}\right]
\end{aligned}
$$

Replacing the summations by integrations, using that

$$
\begin{aligned}
\sum_{\beta \neq 0} \frac{h_{1,2}(\kappa D|\beta|)}{|\beta|} & =\left[\int_{-(1 / 2) \kappa L}^{-(1 / 2) \kappa D} d x+\int_{(1 / 2) \kappa D}^{(1 / 2) \kappa L} d x\right] \frac{h_{1,2}(|x|)}{|x|} \\
& =2 \int_{(1 / 2) \kappa D}^{(1 / 2) \kappa L} d x \frac{h_{1,2}(x)}{x}
\end{aligned}
$$

Eq. (17) leads to

$$
\begin{aligned}
\sum_{\beta \neq \alpha} \mathbf{T}_{s}(D \hat{\mathbf{u}}(\alpha-\beta)) \cdot \mathbf{F}_{\beta}^{h}= & \frac{1}{2 \pi \eta_{0} D}[G(\kappa D, \kappa L) \hat{\mathbf{I}} \\
& +H(\kappa D, \kappa L) \hat{\mathbf{u}} \hat{\mathbf{u}}] \cdot \mathbf{F}_{\alpha}^{h},
\end{aligned}
$$

where

$$
\begin{aligned}
& G(\kappa D, \kappa L)=\int_{(1 / 2) \kappa D}^{(1 / 2) \kappa L} d x \frac{h_{1}(x)}{x} \text { and } \\
& H(\kappa D, \kappa L)=\int_{(1 / 2) \kappa D}^{(1 / 2) \kappa L} d x \frac{h_{2}(x)}{x} .
\end{aligned}
$$

Note that in the absence of screening, where $\kappa \rightarrow 0$,

$$
G(\kappa D, \kappa L)=\frac{1}{2} \ln \{L / D\}=H(\kappa D, \kappa L) \quad \text { (no screening). }
$$

Using the result (19) in Eqs. (15) and (9) for the incident field $\mathbf{u}_{0}$ thus leads to an explicit expression for the hydrodynamic forces on beads,

$$
\begin{aligned}
\mathbf{F}_{\alpha}^{h}= & -\frac{12\left(\pi \eta_{0}\right)^{2} D a}{G(\kappa D, \kappa L)} \\
& \times\left[\hat{\mathbf{I}}-\frac{H(\kappa D, \kappa L)}{G(\kappa D, \kappa L)+H(\kappa D, \kappa L)} \hat{\mathbf{u}} \hat{\mathbf{u}}\right] \cdot \mathbf{T}_{s}(\mathbf{R}-D \alpha \hat{\mathbf{u}}) \cdot \mathbf{v}_{p},
\end{aligned}
$$

where $\mathbf{R}=\mathbf{r}_{p}-\mathbf{r}_{c}$ is the distance between the centers $\mathbf{r}_{p}$ and $\mathbf{r}_{c}$ of the sphere and the rod, respectively. The first term on the left-hand side of the integral equation (15) is logarithmically in $L / D$ smaller than the bead-index sum, and is therefore neglected. From the expression (11) for the reflected flow field and Faxén's theorem (10) it thus finally follows that

$$
\mathbf{F}^{\mathrm{ext}}=6 \pi \eta_{0} a[\hat{\mathbf{I}}+\langle\mathbf{M}(\mathbf{R}, \hat{\mathbf{u}})\rangle] \cdot \mathbf{v}_{p},
$$

with the tensor $\mathbf{M}$ is equal to

$$
\begin{aligned}
\mathbf{M}(\mathbf{R}, \hat{\mathbf{u}})= & \frac{12\left(\pi \eta_{0}\right)^{2} a}{G(\kappa D, \kappa L)} \int_{-(1 / 2) L}^{(1 / 2) L} d l \mathbf{T}_{s}(\mathbf{R}-l \hat{\mathbf{u}}) \cdot\{\hat{\mathbf{I}} \\
& \left.-\frac{H(\kappa D, \kappa L)}{G(\kappa D, \kappa L)+H(\kappa D, \kappa L)} \hat{\mathbf{u}} \hat{\mathbf{u}}\right\} \cdot \mathbf{T}_{s}(\mathbf{R}-l \hat{\mathbf{u}}),
\end{aligned}
$$

where, as before, the bead-index sum is replaced by a contour integral. The brackets $\langle\cdots\rangle$ in Eq. (23) denote ensemble averaging with respect to the degrees of freedom of the sphere and rod.

In order to account for the $N$ rods in the network, Eq. (23) is written as 


$$
\mathbf{F}^{\mathrm{ext}}=6 \pi \eta_{0} a\left[\hat{\mathbf{I}}+\sum_{j=1}^{N}\left\langle\mathbf{M}\left(\mathbf{R}_{j}, \hat{\mathbf{u}}_{j}\right)\right\rangle\right] \cdot \mathbf{v}_{p} .
$$

The assumption here is that flow fields that are reflected by a rod to another rod back to the sphere are relatively weak. As mentioned before, for the open network under consideration, this is probably a good approximation.

The probability density function with respect to which the ensemble average should be evaluated is distorted as a result of the external force on the tracer sphere. This distortion is proportional to the external field, and therefore gives rise to second-order terms in Eq. (25). Since such nonlinear terms are irrelevant for the calculation of the diffusion coefficient, the distortion of the probability density function can be neglected in the evaluation of the hydrodynamic contribution to the diffusion coefficient. The expression (25) can thus simply be averaged with respect to the probability density function (PDF) in the absence of an external force on the sphere. According to Eqs. (1), (2), (24), and (25), this leads to the following expression for the long-time self-diffusion coefficient $D_{s}^{h}$ where only hydrodynamic interactions are accounted for (as indicated by the superscript $h$, while the subscript on $\alpha^{h}$ indicates that its value is different for isotropic and nematic suspensions where either diffusion parallel or perpendicular to the director is considered),

$$
\frac{D_{s}^{h}}{D_{0}}=\frac{1}{1+\alpha_{\mathrm{iso}, \|, \perp}^{h} \varphi},
$$

with $\varphi=(\pi / 4) D^{2} L \bar{\rho}$ the volume fraction of rods $(\bar{\rho}$ the number density of rods), and where the coefficient $\alpha^{h}$ is given by

$$
\begin{aligned}
\alpha_{\mathrm{iso}, \|, \perp}^{h}= & \frac{4 V}{\pi D^{2} L} \hat{\mathbf{v}}_{p} \cdot\left[\int d \mathbf{r}_{p} \int d \mathbf{r}_{c} \oint d \hat{\mathbf{u}}\right. \\
& \left.\times P_{0}\left(\mathbf{r}_{p}, \mathbf{r}_{c}, \hat{\mathbf{u}} \mid \varphi\right) \mathbf{M}(\mathbf{R}, \hat{\mathbf{u}})\right] \cdot \hat{\mathbf{v}}_{p},
\end{aligned}
$$

where $\hat{\mathbf{v}}_{p}=\mathbf{v}_{p} / v_{p}$ is the unit vector along the ensembleaveraged velocity of the tracer particle, and $P_{0}$ is the PDF for the positions $\mathbf{r}_{c}$ and $\mathbf{r}_{p}$ of the center of a rod and of the tracer particle, respectively, and the orientation $\hat{\mathbf{u}}$ of a rod in the network. This PDF depends only on $\mathbf{r}_{c}$ and $\mathbf{r}_{p}$ through the distance $\mathbf{R}=\mathbf{r}_{p}-\mathbf{r}_{c}$ between the sphere and a rod. The subscript " 0 " on $P$ is used to indicate that this is the equilibrium PDF, in the absence of the external force on the sphere. The superscript " $h$ " on $\alpha$ stands for "hydrodynamic," to distinguish it from the coefficient $\alpha$ defined in Ref. 31, which describes the effect of direct interactions. Furthermore, $V$ is the volume of the system under consideration. For a homogeneous network, the PDF $P_{0}$ is simply equal to

$$
P_{0}\left(\mathbf{r}_{p}, \mathbf{r}_{c}, \hat{\mathbf{u}} \mid \varphi\right)=\frac{1}{V^{2}} P_{0}(\hat{\mathbf{u}} \mid \varphi) \exp \{-\beta V(\mathbf{R}, \hat{\mathbf{u}})\},
$$

where $P_{0}(\hat{\mathbf{u}} \mid \varphi)$ is the PDF for the orientation of a rod, $\beta$ $=1 / k_{B} T$ (with $k_{B}$ is Boltzmann's constant and $T$ is the temperature), and $V\left(\mathbf{R}=\mathbf{r}_{p}-\mathbf{r}_{c}, \hat{\mathbf{u}}\right)$ is the pair-interaction potential for a rod and the tracer sphere. Indirect interactions between the sphere and a rod, mediated by surrounding rods, are neglected here. These indirect forces are small for the small tracer sphere in the relatively open network. The expression (27) can thus be written as

$$
\begin{aligned}
\alpha_{\text {iso, }, \|, \perp}^{h}= & \frac{4}{\pi D^{2} L} \hat{\mathbf{v}}_{p} \cdot\left[\int d \mathbf{R} \oint d \hat{\mathbf{u}} P_{0}(\hat{\mathbf{u}} \mid \varphi)\right. \\
& \times \exp \{-\beta V(\mathbf{R}, \hat{\mathbf{u}})\} \mathbf{M}(\mathbf{R}, \hat{\mathbf{u}})] \cdot \hat{\mathbf{v}}_{p} .
\end{aligned}
$$

In the above results, it is assumed that the force on the tracer sphere is colinear with its velocity. This is the case we consider in the experimental section, where diffusion in an isotropic network is considered, as well as diffusion in a nematic rod suspension either parallel or perpendicular to the nematic director. The more general case where the force and velocity are not colinear with the nematic director can be described as a linear combination of the colinear geometries.

For an isotropic network, $P(\hat{\mathbf{u}} \mid \varphi)$ is simply equal to $1 / 4 \pi$. For a nematic network however, this PDF depends on the concentration of rods, since the degree of alignment increases with increasing concentration. The following, reasonably accurate Gaussian representation for this PDF will be used (similar to the Gaussian approximation that Odijk used in Ref. 45 in his treatment of Onsager's prediction ${ }^{46}$ of isotropic-nematic phase transition),

$$
\begin{aligned}
P_{0}(\hat{\mathbf{u}} \mid \varphi)= & \frac{1}{N(C)}\left[\exp \left\{-C \Theta^{2}\right\}\right. \\
& +\exp \left\{-C(\pi-\Theta)^{2}\right], \quad 0 \leqslant \Theta<\pi,
\end{aligned}
$$

where $\Theta$ is the angle between $\hat{\mathbf{u}}$ and the nematic director. The parameter $C$ measures the width of the orientational distribution. Furthermore, $N$ is the normalization constant, which is a unique function of $C$. Note that, for the uniaxial nematic under consideration here, the symmetry condition $\Theta \leftrightarrow(\pi-\Theta)$ is satisfied by the above expression. The constants $N$ and $C$ are determined as functions of the fd concentration from experimental values for the order parameter for $\mathrm{fd}$ virus in $110 \mathrm{mM}$ buffer as a function of concentration, as determined from x-ray scattering data ${ }^{47}$ (see the inset in Fig. $3)$.

Without hydrodynamic screening, the screened Oseen tensor in Eq. (24) for the tensor $\mathbf{M}$ becomes equal to the Oseen tensor, which varies like $\sim R^{-1}$. The integrand in Eq. (29) thus varies like $\sim R^{-2}$ for large distances, so that $\alpha^{h}$ diverges. This was already noted by Altenberger in case of diffusion through a set of fixed point scatterers. ${ }^{21,23}$ The screened Oseen tensor, however, varies like $\sim R^{-3}$ for large distances, which renders $\alpha$ convergent. Hydrodynamic screening is thus an essential feature in describing selfdiffusion through networks of rods. Note that the effective upper limit for the $R$ integration in Eq. (29) is set by the hydrodynamic screening length. The numerical value of diffusion coefficients is thus quite sensitively depending on the precise value of $\kappa$.

Numerical results for the coefficient $\alpha^{h}$ are given in Fig. 3(a) for an isotropic fd network and in Fig. 3(b) for a nematic fd network (length of an fd virus particle is $L=880 \mathrm{~nm}$ and its thickness is $D=6.8 \mathrm{~nm}$ ). The curves in Fig. 3(a) marked with the numbers " 1 " and " 5 " refer to spheres with a diam- 

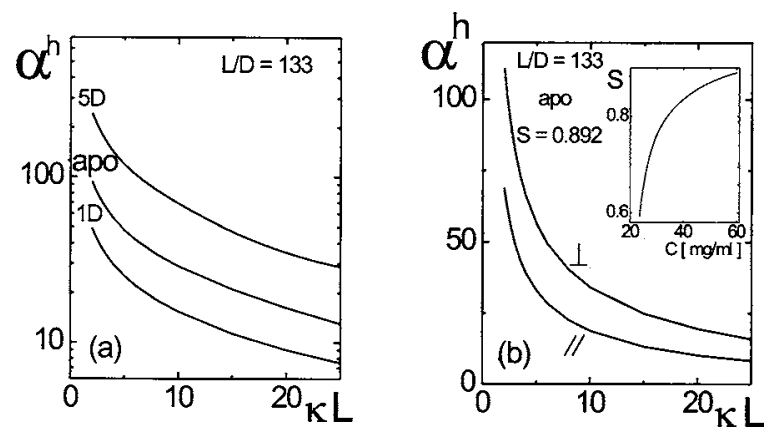

FIG. 3. (a) The coefficient $\alpha^{h}$ as a function of $\kappa L$ for isotropic fd (length of an fd virus particle is $L=880 \mathrm{~nm}$ and its thickness is $D=6.8 \mathrm{~nm}$ ) for three different tracer spheres: "apo" refers to apoferritin which has a diameter of $12.8 \mathrm{~nm}$, while the number $1 D$ and $5 D$ refer to spheres with a diameter of $D$ and 5D, respectively. (b) The coefficient $\alpha^{h}$ for nematic fd and apoferritin. The upper and lower curve are for diffusion perpendicular and parallel to the nematic director, respectively. Here, the order parameter is equal to $S$ $=0.892$, corresponding to the highest concentration of $\mathrm{fd} \mathrm{of} 60 \mathrm{mg} / \mathrm{ml}$ in the experiments. The insert shows the concentration dependence of $S$ as a function of fd concentration for a salt concentration of $110 \mathrm{mM}$, which complies with the experiments described later. The data for the insert are taken from Ref. 47.

eter of $D$ and $5 D$, while the curve marked with "apo" is for apoferritin (diameter, $12.8 \mathrm{~nm}$ ). The coefficient $\alpha^{h}$ decreases with decreasing screening length. The coefficient $\alpha^{h}$ is approximately proportional to the size of the tracer sphere for large screening lengths, which can be seen explicitly from Eq. (24) for the tensor M. As can be seen from Fig. 3(b), the coefficient $\alpha^{h}$ is about a factor of 2 larger for diffusion perpendicular as compared with diffusion parallel to the director. Diffusion is thus predicted to be faster along the nematic director than perpendicular to the director, as far as the hydrodynamic contribution is concerned.

\section{B. The shadowing contribution}

There are two different configurations of the rod relative to the external force acting on the tracer sphere that need to be considered: $\hat{\mathbf{u}} \| \mathbf{F}^{\text {ext }}$ and $\hat{\mathbf{u}} \perp \mathbf{F}^{\text {ext }}$ (as depicted in Figs. 4(a) and 4 (b), respectively). In case $\hat{\mathbf{u}} \| \mathbf{F}^{\text {ext }}$, the pair-correlation

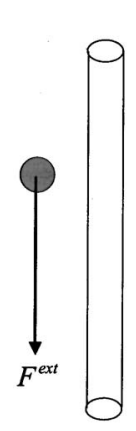

(a)

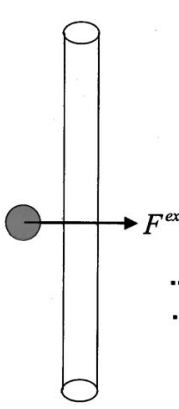

(b)

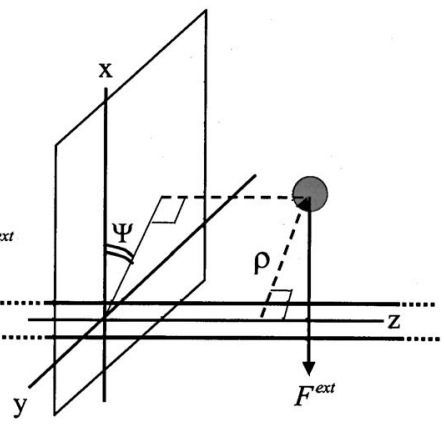

(c)
FIG. 4. The distortion of the pair-correlation function for motion of the sphere parallel to the rods orientation (a) is much less pronounced as for perpendicular motion (b). (c) Definition of the cylindrical coordinates: $\rho$ is the shortest distance between the center line of the rod and the center of the sphere and $\Psi$ is the angle between the projection of the position vector of the sphere onto the $x y$ plane and the positive $x$ axis. Note that the external force is directed along the negative $x$ direction. function is mainly distorted when the sphere interacts with the tips of the rod. In case $\hat{\mathbf{u}} \perp \mathbf{F}^{\mathrm{ext}}$ the distortion is effective over the entire contour of the rod, and is therefore much more pronounced. We shall thus consider the case where $\hat{\mathbf{u}} \perp \mathbf{F}^{\mathrm{ext}}$ and construct the distortion of the pair-correlation function for arbitrary orientations of the rod from these two special cases. Furthermore, since the diameter of the sphere is small compared with the length of the rod, end effects will be neglected, that is, the rod is taken infinitely long.

In the overdamped limit, on the Brownian time scale, inertial forces on the tracer sphere are very small as compared with all other forces. The resulting force balance implies that

$$
\mathbf{F}^{\mathrm{ext}}-\nabla V-k_{B} T \nabla \ln \{g\}+\mathbf{F}^{h}=0,
$$

where $\nabla$ is the gradient operator with respect to the position coordinate of the tracer sphere. Furthermore, $V$ is the potential that is set up by a rod, which is again assumed to be fixed in space due to entanglement forces, $g$ is the pair-correlation function, and $\mathbf{F}^{h}$ is the force with which the fluid acts on the sphere. The third term in Eq. (31) is the Brownian force.

The hydrodynamic force on the tracer sphere has been calculated in the previous subsection as

$$
\mathbf{F}^{h}=-\gamma_{p}^{h} \mathbf{v}, \quad \gamma_{p}^{h}=1+\alpha^{h} \varphi,
$$

where $\mathbf{v}$ is the translational velocity of the sphere and where the coefficient $\alpha^{h}$ is given in Eq. (29). The interpretation in using $\gamma_{p}^{h}$ as a prefactor here is that the sphere moves in an effective Debye-Büche-Brinkman fluid. From the force balance equation (31) it thus follows that,

$$
\mathbf{v}=D_{s}^{h}\left[\beta \mathbf{F}^{\mathrm{ext}}-\beta \nabla V-\nabla \ln \{g\}\right] .
$$

The diffusion coefficient where both hydrodynamic and direct interactions are accounted for follows from Eqs. (1) and (2) by ensemble averaging of Eq. (33),

$$
\mathbf{v}_{p} \equiv\langle\mathbf{v}\rangle=D_{s}^{h}\left[\beta \mathbf{F}^{\mathrm{ext}}-\beta\langle\nabla V\rangle-\langle\nabla \ln \{g\}\rangle\right] .
$$

When the distortion of the pair correlation is neglected, $g$ is equal to

$$
g=\exp \{-\beta V\} \quad \text { (no distortion), }
$$

so that Eq. (34) reduces to

$$
\mathbf{v}_{p}=D_{s}^{h} \beta \mathbf{F}^{\text {ext }} \quad \text { (no distortion), }
$$

which implies that the diffusion coefficient is equal to $D_{s}^{h}$, as it should. Including the distortion of $g$ leads to additional contributions to $D_{s}$ due to direct interactions of the tracer sphere with the rods.

In order to evaluate the ensemble average in Eq. (34), $g$ must be known explicitly. Thus we have to solve the continuity equation,

$$
\frac{\partial g}{\partial t}=\nabla \cdot(\mathbf{v} g)=D_{s}^{h} \nabla \cdot\left[\beta g \mathbf{F}^{\mathrm{ext}}-\beta g \nabla V-\nabla g\right],
$$

where Eq. (33) for the velocity has been used.

Neglecting end effects, the pair-correlation function is a function of the cylindrical coordinates $\Psi$ and $\rho$ [see Fig. 4(c)]. Since we are interested in the linear relationship between the external force on the tracer sphere and the result- 
ing velocity, equations can be linearized with respect to $\mathbf{F}^{\mathrm{ext}}$. The pair-correlation function is therefore written as [with $g_{0}(\rho)=\exp \{-\beta V(\rho)\}$ the undistorted pair-correlation function]

$$
g(\rho, \Psi)=g_{0}(\rho)\left[1+\cos \{\Psi\} L(\rho) F^{\mathrm{ext}}\right] .
$$

Substitution into Eq. (37) and transforming to cylindrical coordinates leads to the following equation for the function $L$ :

$$
\beta \frac{d g_{0}(\rho)}{d \rho}+\frac{d}{d \rho}\left[g_{0}(\rho) \frac{d L(\rho)}{d \rho}\right]-g_{0}(\rho) \frac{L(\rho)}{\rho^{2}}=0 .
$$

In case of hard-core interactions, this equation reduces for $\rho>a+D / 2$ to

$$
\frac{d^{2} L(\rho)}{d \rho^{2}}-\frac{L(\rho)}{\rho^{2}}=0,
$$

since $V(\rho>a+D / 2)=0$, and hence $g_{0}(\rho>a+D / 2)=1$. The solution which vanishes for $\rho \rightarrow \infty$ of this equation is

$$
L(\rho)=A / \rho^{\nu} \quad \text { with } \nu=\frac{1}{2}[1+\sqrt{5}]=1.618 \ldots .
$$

The integration constant $A$ is determined as follows. For vanishing $\epsilon$, again in the case of hard-core interactions (here $\delta$ is the one-dimensional delta distribution), we have

$$
\begin{aligned}
\frac{d g_{0}(\rho)}{d \rho}= & \delta(\rho-a-D / 2) \text { for } \\
& a+D / 2-\epsilon<\rho<a+D / 2+\epsilon \quad(\epsilon \downarrow 0) .
\end{aligned}
$$

Integrating the differential equation (39) from $\rho=a+D / 2$ $-\epsilon$ to $\rho=a+D / 2+\epsilon$ and taking the limit of vanishing $\epsilon$ readily gives

$$
\beta+\frac{d L(\rho=a+D / 2)}{d \rho}=0,
$$

from which it follows that

$$
A=\frac{\beta}{\nu}[a+D / 2]^{1+\nu} .
$$

According to Eq. (38) the distorted pair correlation thus reads

$$
g(\rho, \Psi)=g_{0}(\rho)\left[1+\beta \cos \{\Psi\} \frac{[a+D / 2]^{1+\nu}}{\nu \rho^{\nu}} F^{\mathrm{ext}}\right] .
$$

Using this expression for the pair-correlation function, the ensemble averages on the right-hand side of Eq. (34) can be calculated. Multiplying the resulting "shadowing friction" due to the presence of a single rod with the number of rods in the system one finds

$$
\begin{aligned}
& \beta\langle\nabla V\rangle=\frac{1}{\nu}\left(1+\frac{2 a}{D}\right)^{2} \varphi \beta \mathbf{F}^{\mathrm{ext}}, \\
& \langle\nabla \ln \{g\}\rangle=0 .
\end{aligned}
$$

Mathematical details of the derivation of this result are given in Appendix B.

The above calculation has been done for a rod with a fixed orientation perpendicular to the direction of the external force [see Fig. 4(a)]. For orientations parallel to the ex- ternal force [see Fig. 4(b)] the "shadowing forces" are expected to be much smaller. To within linear response in the external force, which is sufficient for the calculation of the diffusion coefficient, the ensemble-averaged forces can be written as a linear combination due to the component $\hat{\mathbf{u}} \hat{\mathbf{u}} \cdot \mathbf{F}^{\text {ext }}$ of the external force parallel to the rod and the component $[\hat{\mathbf{I}}-\hat{\mathbf{u}} \hat{\mathbf{u}}] \cdot \mathbf{F}^{\text {ext }}$ perpendicular to the rod. Hence, in Eq. (46), the external force can simply be replaced by the component of the external force perpendicular to the rod's orientation and averaged over the orientation of the rod,

$$
\beta\langle\nabla V\rangle=\frac{1}{\nu}\left(1+\frac{2 a}{D}\right)^{2} \varphi \oint d \hat{\mathbf{u}} P_{0}(\hat{\mathbf{u}})[\hat{\mathbf{I}}-\hat{\mathbf{u}} \hat{\mathbf{u}}] \cdot \beta \mathbf{F}^{\mathrm{ext}},
$$

where, as before, $P_{0}$ is the probability density function of the orientation of a rod. Introducing the tensor

$$
\mathbf{Q} \equiv \frac{3}{2}\left\langle\hat{\mathbf{u}} \hat{\mathbf{u}}-\frac{1}{3} \hat{\mathbf{I}}\right\rangle \equiv \frac{3}{2} \oint d \hat{\mathbf{u}} P_{0}(\hat{\mathbf{u}})\left[\hat{\mathbf{u}} \hat{\mathbf{u}}-\frac{1}{3} \hat{\mathbf{I}}\right],
$$

Eq. (48) can be written as

$$
\beta\langle\nabla V\rangle=\frac{2}{3 \nu}\left(1+\frac{2 a}{D}\right)^{2} \varphi[\mathbf{I}-\mathbf{Q}] \cdot \beta \mathbf{F}^{\mathrm{ext}},
$$

For an isotropic network, $\mathbf{Q}=0$. For a nematic network, and for diffusion parallel to the director where $\mathbf{F}^{\text {ext }}$ is colinear with the director, $\mathbf{Q} \cdot \mathbf{F}^{\text {ext }}=S \mathbf{F}^{\text {ext }}$, with $S$ the largest eigenvalue of $\mathbf{Q}\left(S\right.$ is sometimes alternatively denoted as $\left.P_{2}\right)$. For the uniaxial nematic under consideration, when $\mathbf{F}^{\text {ext }}$ is perpendicular to the director, $\mathbf{Q} \cdot \mathbf{F}^{\mathrm{ext}}=-\frac{1}{2} S \mathbf{F}^{\mathrm{ext}}$. From the above expression (49) and Eq. (34) it thus follows that the selfdiffusion coefficient $D_{s}$, where both hydrodynamic interactions and shadowing are included, is equal to

$$
\frac{D_{s}}{D_{0}}=\frac{D_{s}^{h} D_{s}^{s}}{D_{0}^{2}}
$$

where, as before, $D_{s}^{h}=k_{B} T / \gamma^{h}$ is the diffusion coefficient where only hydrodynamics is accounted for, and $D_{s}^{s}$ is equal to

$$
\frac{D_{s}^{s}}{D_{0}}=1-\alpha_{\text {iso }, \|, \perp}^{s} \varphi,
$$

where

$$
\begin{aligned}
& \alpha_{\text {iso }}^{s}=\frac{2}{3 \nu}\left(1+\frac{2 a}{D}\right)^{2}, \\
& \alpha_{\|}^{s}=\frac{2}{3 \nu}\left(1+\frac{2 a}{D}\right)^{2}(1-S), \\
& \alpha_{\perp}^{s}=\frac{2}{3 \nu}\left(1+\frac{2 a}{D}\right)^{2}\left(1+\frac{1}{2} S\right),
\end{aligned}
$$

for diffusion in an isotropic network and a nematic network parallel and perpendicular to the director, respectively. The diffusion coefficient $D_{s}^{s}$ is the diffusion coefficient that incorporates shadowing (the superscript $s$ stands for "shadowing"). Since direct interactions with just a single rod are independently added, the results in Eqs. (51) and (52) must be 
regarded as the first term in an expansion with respect to the volume fraction $\varphi$ of rods.

For the fd networks for which experiments will be discussed later in this paper, typical volume fractions are of the order 0.01. Putting in numbers in the numerators in Eq. (51) with $2 a / D$ of order 1 then reveals that the shadowing effect is very small. For larger tracer spheres, for which typically $2 a / D=10$, the shadowing effect becomes relevant.

\section{Theoretical summary}

It follows from Eqs. (50), (26), and (51) that the selfdiffusion coefficient $D_{s}$, where both hydrodynamic interactions and shadowing are accounted for, is equal to

$$
\frac{D_{s}}{D_{0}}=\frac{1}{1+\alpha_{\mathrm{iso}, \|, \perp}^{h} \varphi} \frac{1}{1+\alpha_{\mathrm{iso}, \|, \perp}^{s} \varphi} .
$$

Here, the coefficient $\alpha^{h}$ accounts for screened hydrodynamic interactions and $\alpha^{s}$ accounts for the shadowing effect. The coefficient $\alpha^{h}$ is given in Eq. (29), which value depends on whether an isotropic or nematic network with diffusion parallel or perpendicular to the director is considered. The coefficient $\alpha^{s}$ is given in Eq. (52). Here, we have written 1 $-\alpha^{s} \varphi$ for convenience as $1 /\left(1+\alpha^{s} \varphi\right)$, which is allowed due to the small numerical value of $\alpha^{s} \varphi$. The main rodconcentration dependence of the self-diffusion coefficient originates from hydrodynamic interactions.

The hydrodynamic-interaction coefficient $\alpha^{h}$ depends on the aspect ratio $p=L / D$ of the rods, the size ratio $q=2 a / L$ of the diameter of the tracer sphere and the length of the rods, and on the orientational order parameter $S$ of the network through the orientational probability density function in Eq. (29). In addition, $\alpha^{h}$ depends sensitively on the screening length $1 / \kappa$. Hence,

$$
\alpha_{\mathrm{iso}, \|, \perp}^{h} \equiv \alpha^{h}\left(p, q, \kappa^{-1} / L, S\right) .
$$

The shadowing coefficient $\alpha^{s}$ is, according to Eq. (52) a function of $2 a / D=q / p$ and $S$,

$$
\alpha_{\text {iso, }, \|, \perp}^{s} \equiv \alpha^{s}(q / p, S) .
$$

The parameters $p$ and $q$ as well as the orientational order parameter $S$ can be measured independently. The unknown quantity is the hydrodynamic screening length.

As far as we know, there is no independent theory for the screening length of networks of very long and thin rods. As a first attempt one might consider a self-consistent calculation of the screening length, which is given in Appendix C. It turns out that at a somewhat higher fd concentration, the self-consistent screening length becomes of the order of the thickness of the rod, where the concept of screening breaks down. Comparing the above theory directly with experiments, however, leads to much larger screening length (see Sec. IV). The probable reason for the failure of a selfconsistent calculation is probably that the hydrodynamic field that a rod experiences is never homogeneous over its whole length. A simple calculation of the friction coefficient of a rod in an otherwise quiescent (screened) fluid is therefore unrealistic.
The most severe approximation for nematic networks is the neglect of the anisotropy of screening. The argument given before is that hydrodynamic interactions are most pronounced in directions in which the tracer sphere is pulled through the network. To really quantify the effect of anisotropic screening, the corresponding Debye-Büche-Brinkman equation should be solved explicitly. So far this has not been done.

Finally we will show that the long-time self-diffusion coefficient is relevant for the mass transport of small spheres through a network, provided that the network is not distorted by the presence of the small sphere. Consider a homogeneous suspension of rods in which spherical particles are dispersed with a small concentration gradient. When the concentration of dispersed spheres is very small, so that they do not interact with each other, the only force a tracer sphere experiences is the Brownian force, $-k_{B} T \nabla \rho(\mathbf{r}, t)$ (where $k_{B}$ is Boltzmann's constant, $T$ is the temperature, and $\rho(\mathbf{r}, t)$ is the number concentration of spheres at point $\mathbf{r}$ at time $t$ ). Replacing the external force in Eq. (2) by the Brownian force, the ensemble-averaged translational velocity $\mathbf{v}_{p}(\mathbf{r}, t)$ of a sphere at position $\mathbf{r}$ at time $t$ is thus found to be equal to, $\mathbf{v}_{p}(\mathbf{r}, t)$ $=-D_{s} \nabla \rho(\mathbf{r}, t)$. Substitution of this result into the continuity equation, $\partial \rho(\mathbf{r}, t) / \partial t=-\nabla \cdot\left[\mathbf{v}_{p}(\mathbf{r}, t) \rho(\mathbf{r}, t)\right]$, immediately leads to Fick's diffusion equation,

$$
\frac{\partial}{\partial t} \rho(\mathbf{r}, t)=D_{s} \nabla^{2} \rho(\mathbf{r}, t) \text {. }
$$

The gradient diffusion constant for mass transport of tracer spheres in homogeneous networks of rods is thus equal to the long-time self-diffusion coefficient of the spheres. This result relies on the assumption that the rod concentration is uniform. The long-time self-diffusion coefficient is thus relevant for mass transport of spheres through homogeneous networks of rods.

\section{THE COLLOIDAL SYSTEMS AND SAMPLE PREPARATION}

The bacteriophage fd is a rodlike molecule with a contour length of $L=880 \mathrm{~nm}$, a bare diameter of $D=6.6 \mathrm{~nm}$, a persistence length of $P=2200 \mathrm{~nm}$, a molecular weight of $M=1.64 \times 10^{7} \mathrm{~g} / \mathrm{mol}$, and a maximum charge density of $10 \mathrm{e} / \mathrm{nm}$ in water at $\mathrm{pH}=8.15$. $^{48}$ The fd virus was grown and purified following standard biological protocols ${ }^{49}$ using the XL1blue strain of E. coli as the host bacteria. The standard yield is ca. $15 \mathrm{mg}$ of fd per liter of infected bacteria, and virus is typically grown in 61 batches. The virus particles were finally purified by repeated centrifugation $\left(10^{5} \mathrm{~g}\right.$ for $5 \mathrm{~h}$ ) and redispersed in a $20 \mathrm{mM}$ tris- $\mathrm{HCl}$ buffer at $p \mathrm{H} 8.15$ with $100 \mathrm{mM} \mathrm{NaCl}$ to screen the electrostatic interactions. The buffer contributes about $10 \mathrm{mM}$ to the total ionic strength.

Apoferritin is used as a tracer sphere. Ferritin is an iron storage protein, which is found in a variety of animals. It consists of a protein shell surrounding a ferrous core. This protein shell of ferritin is called apoferritin [molecular weight (MW), 450-475 kD]. Apoferritin consists of 24 peptide subunits joined through noncovalent interactions. The 


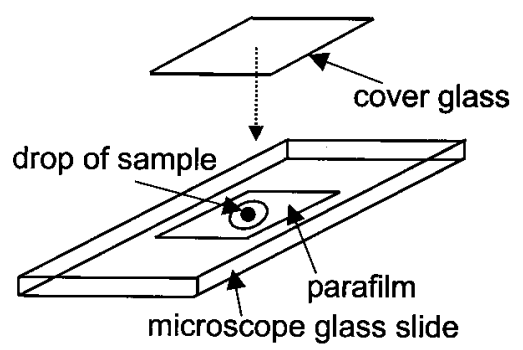

FIG. 5. Construction of the cuvette which enables FCS measurements of nematic samples. The construction is described in detail in the main text.

apoferritin shell carries a net negative charge at neutral $p \mathrm{H}$ which ensures its excellent solubility in water. From the point of view of colloid science, apoferritin is a very nice monodisperse model system of small and spherically shaped particles which, under proper conditions, can acquire a high surface charge density. Apoferritin solutions are perfectly monodisperse and relatively stable, i.e., for weeks at room temperature and even for months at $4{ }^{\circ} \mathrm{C}$. Apoferritin from horse spleen was purchased from SIGMA. The concentration of this sample was approximately $51 \mathrm{mg} / \mathrm{ml}$, the sodium salt content was approximately $100 \mathrm{mM}$. Apoferritin was fluorescently labeled with 5(6) TAMRA-SE [5(6) carboxytetramethylrhodamine, succinimidyl ester]-Sigma-Aldrich, Product number C4759, Lot 100k1600. The dye was connected to the $N$ terminus of the protein chain using a standard procedure. After labeling, apoferritin-TAMRA was purified from the free dye by dialysis using $10 \mathrm{kD}$ filters (millipore "ultrafree") and centrifuged at $7500 \mathrm{~g}$. There are several literature values for the size of apoferritin (see, for example, Refs. 50-55). From these literature values we decided to use in our calculations a diameter of $12.8 \mathrm{~nm}$. Literature values vary within about $\pm 1 \mathrm{~nm}$ from this value.

In order to be able to perform FCS measurements on nematic samples, homemade cuvettes were used with thicknesses of 100-120 $\mu \mathrm{m}$. This ensures that the nematic director can be kept uniform over the thickness of the sample during a FCS measurement. The optical quality of capillaries turned out to be not good enough to ensure reproducible confocal volumes. Cover-glass optical quality is needed to render the confocal volume appropriately defined. A piece of parafilm with a circular hole in the middle of about $7 \mathrm{~mm}$ diameter was firmly pressed onto a microscope glass slide, as depicted in Fig. 5. The parafilm has thicknesses of $100-120 \mu \mathrm{m}$. A drop of the sample with a volume of about $2 \mu \mathrm{l}$ is then carefully positioned in the middle of the circular hole. The sample should not touch the parafilm. A cover glass with a thickness of about $80 \mu \mathrm{m}$ is then pressed upon this drop and the surrounding parafilm. The drop touches the cover glass, so that the sample volume is a circular slice of liquid confined between two glass surfaces. The cover glass is hold in place by tape that is wrapped around the sides of the microscope glass slide and the cover glass. During a measurement, the cover glass is on the down side, where the objective of the confocal microscope is positioned. We used a water immersion objective $(63 \times / 1.2 \mathrm{~W}$ Korr Water Immersion Zeiss) and focused from below into the middle of the sample. Other methods that we tried to ensure a stable drop position failed. In obtaining data for the nematic state, the sample was exposed to a magnetic field of about $2 \mathrm{~T}$ for 10 min just before a FCS measurement. The polydomain structure of the sample is then transformed to a monodomain over the entire sample volume. The nematic director is chosen parallel to the cover glass. The measurement is done in the absence of the magnetic field, where the monodomain structure survives for about $1 / 2 \mathrm{~h}$. The degree of orientational order is not affected by the magnetic pretreatment of the sample, since orientational order relaxes relatively fast as a result of local orientational diffusion of rods. The orientation of the nematic director relative to the confocal volume is depicted in Fig. 7.

\section{EXPERIMENTAL RESULTS}

In this section, FCS results will be presented together with a calculation of the concentration dependence of the hydrodynamic screening length from the experimental data using the theory described above. It is a nontrivial matter to extract diffusion coefficients from FCS data of apoferritin in fd suspensions, since fd-virus particles fluoresces by itself to some extent. Section IV A describes the procedure used to extract diffusion coefficients from FCS data on these mixtures, both for isotropic and nematic fd suspensions. In Sec. IV $\mathrm{B}$ the experimental data for the long-time self-diffusion coefficient of apoferritin are presented and the concentration dependence of the hydrodynamic screening length is determined from these data using the theory described above.

\section{A. Fluorescence correlation spectroscopy (FCS) on tracer spheres in isotropic and nematic fd-virus suspensions}

The standard form of a correlation function $C_{f}(t)$ measured with FCS from a single-component solution of small spherical particles, at times larger than about $10 \mu \mathrm{s}$ where triplet contributions are absent, reads

$$
C_{f}^{0} \sim\left[1+\frac{D_{s} t}{\sigma_{1}^{2}}\right]^{-1}\left[1+\frac{D_{s} t}{\sigma_{2}^{2}}\right]^{-1 / 2},
$$

where $D_{s}$ is the long-time self-diffusion coefficient of the spherical particles. The superscript 0 refers to the standard form. Furthermore $\sigma_{1}$ and $\sigma_{2}$ are the linear dimensions of the confocal volume, perpendicular and parallel to the propagation direction of the laser beam. These dimensions are defined through the Gaussian intensity distribution $I_{0}(\mathbf{r})$ within the confocal volume as

$$
I_{0}(\mathbf{r}) \sim \exp \left\{-\left(x^{2}+y^{2}\right) / 2 \sigma_{1}^{2}\right\} \exp \left\{-z^{2} / 2 \sigma_{2}^{2}\right\},
$$

where the $z$ direction is chosen as the propagation direction of the laser beam. Typically, $\sigma_{1} \approx 200 \mathrm{~nm}$ and $\sigma_{2} \approx 900 \mathrm{~nm}$.

For later reference, we shall rewrite Eq. (56) as

$$
C_{f}^{0}(t \mid \tau) \sim\left[1+\frac{t}{\tau}\right]^{-1}\left[1+\frac{1}{S^{2}} \frac{t}{\tau}\right]^{-1 / 2},
$$

where the relaxation time $\tau$ is equal to 


$$
\tau=\sigma_{1}^{2} / D_{s}
$$

and the so-called structure parameter $S=\sigma_{2} / \sigma_{1}$ is found from measurements on solutions of free rhodamine to be equal to

$$
S=\sigma_{2} / \sigma_{1}=4.80 \pm 0.05
$$

Note that the last factor in Eq. (58) relates to diffusion in the propagation direction of the laser beam, while the first factor is related to diffusion in the two perpendicular directions. For a mixture of small spherical, fluorescent, noninteracting spheres, the measured fluorescence correlation function is the sum of functions of the form (56), with the corresponding relaxation times for the various species.

When the fluorescent spherical particles are not very small compared with the linear dimensions of the confocal volume, both $\sigma_{1}$ and $\sigma_{2}$ should be replaced by larger "effective" dimensions, which will change the value of the structure parameter. ${ }^{31}$ This is due to the fact that when the rim of a particle enters the confocal volume, but its center is still outside, it will already contribute to the detected fluorescent intensity. The apoferritin particles used in the present study are small enough to neglect this effective increase of the confocal dimensions.

A complication with FCS experiments on mixtures of proteins and fd virus is that the nonspherical fd-virus particles themselves contribute slightly to the fluorescent intensity. The many proteins attached to the fd-virus particle probably fluoresce to some extent. In addition, a small amount of molecular impurity is present in our fd suspensions that contributes a small fraction to the total intensity. These two contributions have to be accounted for when interpreting FCS data on tracer solutions of protein in host suspensions of $\mathrm{fd}$ virus. Depending on whether the fd suspension is isotropic or nematic, the contributions other than that arising from the tracer protein are different. We shall discuss both in the following subsections.

\section{Isotropic suspensions}

The molecular impurity in our fd suspensions mentioned above can be simply accounted for by an additional term in the correlation function of the standard form $C_{f}^{0}\left(t \mid \tau_{i}\right)$ in Eq. (58), where $\tau_{i}$ is the relaxation time of the impurity. The contribution of fd-virus particles is much more complicated for two reasons. First of all, fd virus is a nonspherical particle with a length $(1 \mu \mathrm{m})$ that is larger than the linear dimensions of the confocal volume, so that rotational dynamics might affect the fluorescence correlation function. Secondly, the effective linear dimensions of the confocal volume are larger than its geometrical dimensions, since again, fd virus is longer than the linear dimensions of the confocal volume. The effective structure parameter is not known. Correlation functions for fd solutions (without protein) can be written as a sum of the standard contribution from the molecular impurity and a contribution from fd particles (the superscript $b$ stands for background and the subscript $i$ stands for impurity),
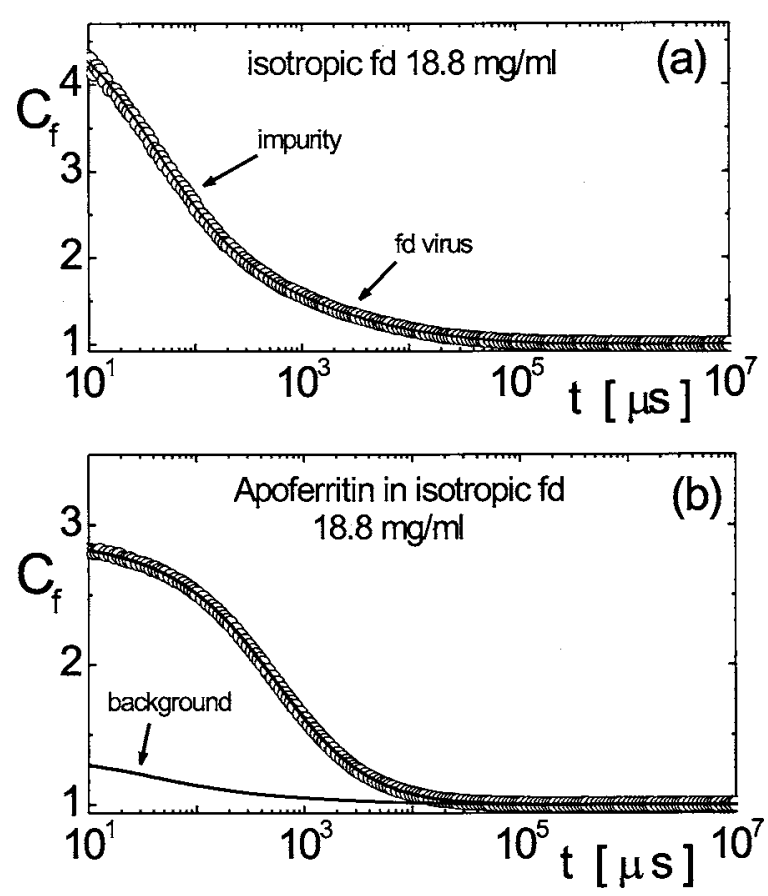

FIG. 6. Typical fluorescence correlation functions for pure isotropic $\mathrm{fd}$ at a concentration of $18.8 \mathrm{mg} / \mathrm{ml}$ (a) and for the mixture of apoferritin and isotropic fd virus (b). The solid curves are fits to the functions described in the main text. The solid curve marked "background" in (b) is the absolute contribution of the pure fd correlation function as plotted in (a) to the correlation function for the mixture.

$$
C_{f}^{b}\left(t \mid A, \tau_{1}, \tau_{2}, \tau_{i}\right) \sim A C_{f}^{\mathrm{fd}}\left(t \mid \tau_{1}, \tau_{2}\right)+(1-A) C_{f}^{0}\left(t \mid \tau_{i}\right)
$$

where $A$ is the contribution of $\mathrm{fd}$ particles relative to the molecular impurity. The following function for the fd contribution to the background correlation function was found to fit experimental data to high accuracy,

$$
C_{f}^{\mathrm{fd}}\left(t \mid \tau_{1}, \tau_{2}\right) \sim\left[1+\frac{t}{\tau_{1}}\right]^{-1 / 2}\left[1+\frac{t}{\tau_{2}}\right]^{-1} .
$$

This is the standard form of the correlation function, where formally, the structure parameter is left as an adjustable parameter. Although there is no clear physical interpretation of the relaxation times $\tau_{1,2}$, these times probably incorporate both translational and rotational dynamics of fd particles as well as the effective structure parameter. The representation (62) of the contribution of fd particles to the measured correlation function is merely of practical importance in order to extract the protein contribution from experimental correlation functions. An example of a fit of the correlation function to Eq. (61) is given in Fig. 6(a). Here, the concentration of fd-virus particles is $18.8 \mathrm{mg} / \mathrm{ml}$ (the isotropic-nematic binodal concentration is $20 \mathrm{mg} / \mathrm{ml}$ ). As can be seen, the fit is very accurate, and will turn out to be sufficient to extract diffusion data for proteins from their mixtures with $\mathrm{fd}$. The fit for this particular concentration gives $A=0.21, \tau_{i}$ $=38.5 \mu \mathrm{s}, \tau_{1}=600 \mu \mathrm{s}$, and $\tau_{2}=7.0 \times 10^{4} \mu \mathrm{s}$. The relaxation time of the impurity is indeed of the order of what one expects for a small molecule (for free rhodamine the relaxation time is equal to $25 \mu \mathrm{s}$ ).

The correlation function for suspensions with a tracer amount of protein is now given by 


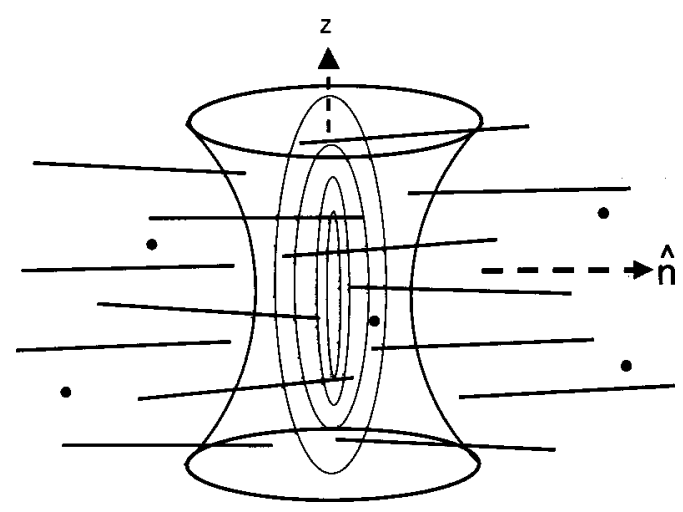

FIG. 7. The orientation of the nematic director $\hat{\mathbf{n}}$ relative to the confocal volume. The propagation direction of the laser beam is along the $z$ axis.

$$
C_{f}(t)=1+\frac{1}{N}\left\{A_{p} C_{f}^{0}\left(t \mid \tau_{p}\right)+\left(1-A_{p}\right) C_{f}^{b}\left(t \mid A, \tau_{1}, \tau_{2}, \tau_{i}\right)\right\}
$$

where $A_{p}$ and $\tau_{p}$ are the relative contribution of the tracer sphere (protein) and its relaxation time, respectively. Here we added the appropriate base line and the amplitude which is related to the average number of particles $N$ within the confocal volume. Once $\tau_{p}$ is obtained, the long-time selfdiffusion coefficient $D_{s}$ relative to the Einstein diffusion coefficient $D_{0}$ is calculated from

$$
D_{s} / D_{0}=\tau_{p}^{0} / \tau_{p}
$$

where $\tau_{p}^{0}$ is the relaxation time for dilute solutions of protein without fd virus as obtained from FCS data fitted to the standard form (56).

The quantity $\tau_{p}$ of interest is thus measured as follows. First determine the parameters $A, \tau_{1}, \tau_{2}$, and $\tau_{i}$, by fitting correlation functions for fd suspensions (without protein) to Eqs. (61) and (62). Add a very small amount of protein and then fit the correlation function of the mixture to Eq. (63) with respect to the parameters $A_{p}$ and $\tau_{p}$, keeping $A, \tau_{1}, \tau_{2}$, and $\tau_{i}$ fixed to the earlier determined values.

A correlation function with a tracer amount of apoferritin is given in Fig. 6(b), again for $18.8 \mathrm{mg} / \mathrm{ml}$, of fd. This result is obtained by averaging ten correlation functions collected during $90 \mathrm{~s}$. The relative contribution of protein is typically $A_{p}=0.80-0.95$. The solid line indicates the background contribution to the correlation function from pure fd, as plotted in Fig. 6(a). At least four of these results are averaged to give the final values for diffusion coefficients reported in the present paper to within an accuracy of about $10 \%$.

\section{Nematic suspensions}

In our experiments the director is perpendicular to the propagation direction of the laser beam, as depicted in Fig. 7. Hence, motion parallel to the director occurs only along the smaller dimension $\sigma_{1}$ of the confocal volume, with the corresponding diffusion coefficient $D_{s, \|}$. Motion perpendicular to the director occurs along the second smaller dimension and the larger dimension $\sigma_{2}$ of the confocal volume, with the
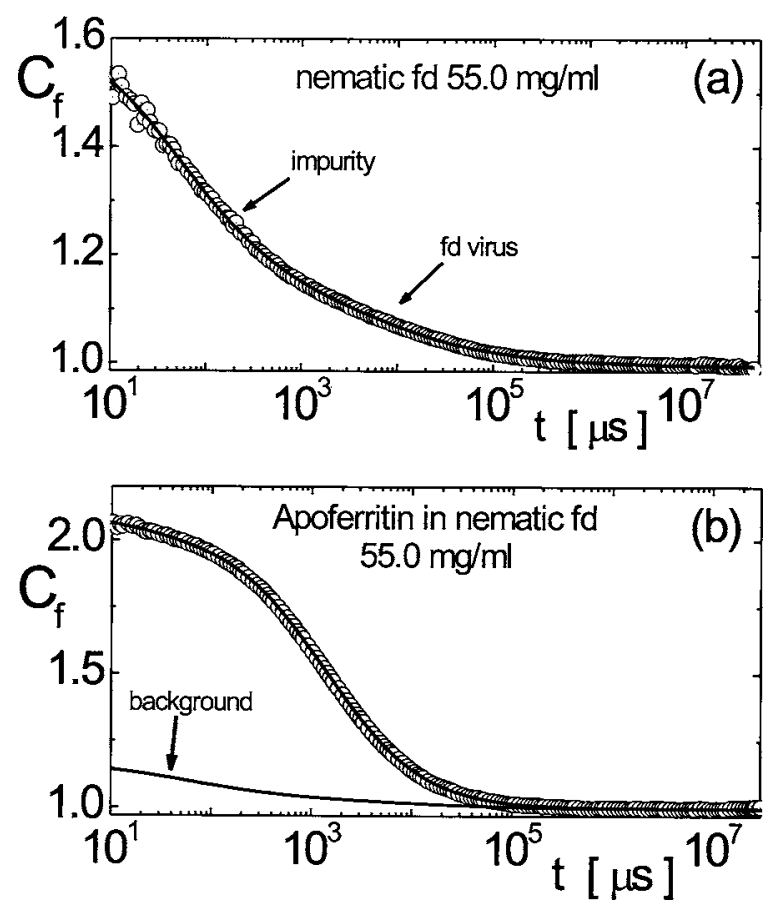

FIG. 8. Typical fluorescence correlation functions for pure nematic fd at a concentration of $55.0 \mathrm{mg} / \mathrm{ml}$ (a) and for the mixture of apoferritin and nematic fd virus corresponding to diffusion perpendicular to the director (b). The solid curves are fits to the functions described in the main text. The solid curve marked background in (b) is the absolute contribution of the pure fd correlation function in (a) to the correlation function for the mixture.

corresponding diffusion coefficient $D_{s, \perp}$. For diffusion of a tracer in a nematic suspension, the standard form (56) thus changes to

$$
C_{f}^{0} \sim\left[1+\frac{D_{s, \|} t}{\sigma_{1}^{2}}\right]^{-1 / 2}\left[1+\frac{D_{s, \perp} t}{\sigma_{1}^{2}}\right]^{-1 / 2}\left[1+\frac{D_{s, \perp} t}{\sigma_{2}^{2}}\right]^{-1 / 2},
$$

which is rewritten, analogous to its isotropic counterpart (62) as

$$
C_{f}^{0}\left(t \mid \tau_{\|}, \tau_{\perp}\right) \sim\left[1+\frac{t}{\tau_{\|}}\right]^{-1 / 2}\left[1+\frac{t}{\tau_{\perp}}\right]^{-1 / 2}\left[1+\frac{1}{S^{2}} \frac{t}{\tau_{\perp}}\right]^{-1 / 2},
$$

where the relaxation times are equal to

$$
\tau_{\perp}=\sigma_{1}^{2} / D_{s, \perp} \quad \text { and } \tau_{\|}=\sigma_{1}^{2} / D_{s, \|} \cdot
$$

Both contributions to the correlation function for the impurity and the protein are given by this expression with the appropriate relaxation times $\tau_{i, \perp, \|}$ and $\tau_{p, \perp, \|}$, respectively.

The contribution of $\mathrm{fd}$ to the correlation function is found to be accurately described by the same function (62) as for isotropic suspensions. The fd contribution is thus characterized by two relaxation times $\tau_{1}$ and $\tau_{2}$. Again, an interpretation of this representation of the correlation function of pure fd in terms of physically meaningful quantities would require a separate study. An example of a fit is given in Fig. 8 (a). Here the concentration of $\mathrm{fd}$ is $55.0 \mathrm{mg} / \mathrm{ml}$, which is far above the concentration of the nematic-isotropic binodal (which is located at about $22 \mathrm{mg} / \mathrm{ml}$ ). The values for the 


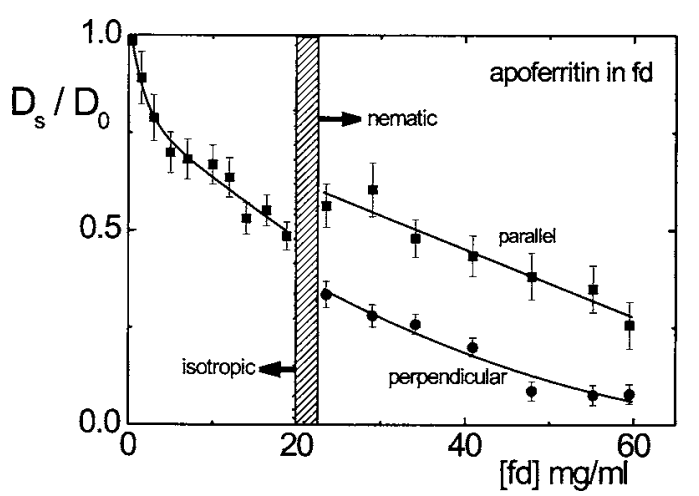

FIG. 9. The self-diffusion coefficient of apoferritin in fd-virus networks in the isotropic state and the nematic state, both along and perpendicular to the nematic director as indicated in the figure. The curves are best sigmoidal fits. The vertical dashed area indicates the isotropic-nematic two-phase region.

different parameters are, $A=0.17, \quad \tau_{i, \perp}=1040 \mu \mathrm{s}, \quad \tau_{i, \|}$ $=24.7 \mu \mathrm{s}, \tau_{1}=7800 \mu \mathrm{s}$, and $\tau_{2}=1.3 \times 10^{5} \mu \mathrm{s}$.

An experimental correlation function with a tracer amount of apoferritin is given in Fig. 8(b), together with the fit to Eq. (63) with the appropriate nematic correlation functions as described above. The correlation function is the average of ten measurements each collected during $90 \mathrm{~s}$. The fd concentration here is $55.0 \mathrm{mg} / \mathrm{ml}$. The solid line in Fig. 8(b) indicates the background contribution from pure fd, as plotted in Fig. 8(a). The relative contribution of protein is typically $A_{p}=0.80-0.95$. Protein relaxation times are obtained in the same way as described in the previous subsection for isotropic suspensions.

\section{B. Diffusion coefficients and screening lengths}

The experimental long-time self-diffusion coefficient of apoferritin, as obtained with FCS as a function of the fdvirus concentration is given in Fig. 9. The vertical dashed area indicates the isotropic-nematic two-phase region. The solid lines are sigmoidal fits to the data points. As can be seen, the diffusion coefficient at the highest concentration in the isotropic fd network is in between the diffusion coefficients for diffusion parallel and perpendicular to the director at the lowest concentration in the nematic network. Diffusion parallel to the director is faster than diffusion perpendicular to the director. The same trend has been found in Fig. 3(b), where the theoretical prediction for the numerical value of the coefficient $\alpha_{\perp}^{h}$ is typically larger than $\alpha_{\|}^{h}$. The same holds for the corresponding shadowing coefficients in Eq. (52). As mentioned before, typical contributions due to shadowing effect to the concentration dependence of the diffusion coefficient are of the order of $1 \%-2 \%$, and are much smaller than the hydrodynamic contribution.

Note that in the isotropic state, the mesh-size $\xi$ of the fd network can be obtained from ${ }^{56}$

$$
\xi=L \sqrt{c^{\star} / c},
$$

where $c$ is the concentration and $c^{\star}=0.076 \mathrm{mg} / \mathrm{ml}$ is the overlap concentration of fd virus. Even for the highest concentrations considered here, the mesh size is considerably larger than the diameter of the apoferritin tracer particles,

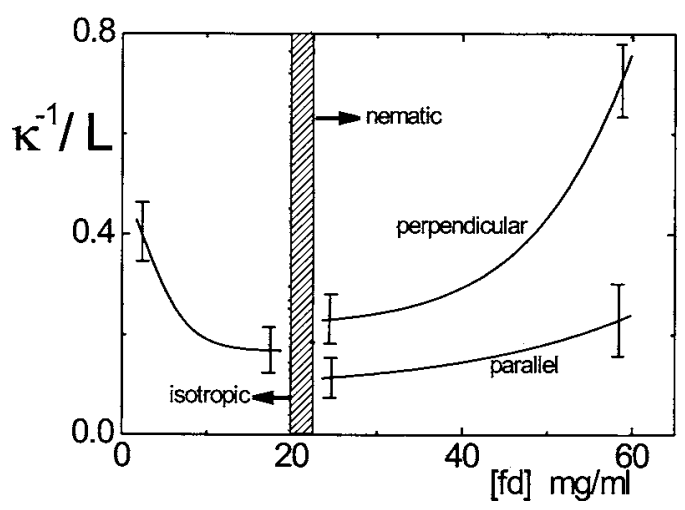

FIG. 10. The hydrodynamic screening length $\kappa^{-1}$ in units of the rod length $L$ as a function of fd concentration in (a) the isotropic fd network and (b) the nematic network, both in the direction parallel and perpendicular to the director, as indicated in the figure. The vertical dashed area indicates the isotropic-nematic two-phase region. The curves are obtained by comparing the best fits in Fig. 9 to theory. The error bars indicate the uncertainty of the screening lengths obtained in this way.

which validates the approximations related to an open network made in the theory in Sec. II.

The hydrodynamic screening length is now obtained from a comparison of the experimental data in Fig. 9 with the theoretical predictions in Eqs. (53), (29), and (52). For each concentration of fd virus, the screening length is chosen such that the theoretical results for the diffusion coefficient coincide with the experimental data. The solid lines in Fig. 10 thus correspond to the solid lines in Fig. 9. The error bars in Fig. 10 are estimated errors in the screening length complying with the experimental error bars in Fig. 9. The screening length in the isotropic fd networks decreases with increasing fd concentration, as expected, and seems to level off to a constant at higher concentrations. For neniatic networks however, the screening length increases with increasing fd concentration. This counterintuitive result is due to the increasing degree of orientational order on increasing the fd concentration. Increasing the rod concentration and keeping the degree of orientational order fixed would lead to a shorter screening length. Apparently, the increase of the screening length due to an increase in orientational order is stronger than its decrease due to an increase of concentration. This phenomenon is also exhibited by the shear viscosity of lyotropic nematics: the shear viscosity, on first thought unexpectedly, decreases on increasing the concentration of a nematic suspension due to the increase of orientational order (see, for example, Refs. 57 and 58).

There is as yet no independent theory for the calculation of the hydrodynamic screening length. The self-consistent calculation of the screening length as given in Appendix C only works at low rod concentration but fails at higher concentration. The probable reason for this failure is that the hydrodynamic field that a rod experiences is not homogeneous over its whole length.

\section{SUMMARY AND CONCLUSION}

For diffusion of tracer spheres in networks of rods, there are two extreme cases to be distinguished, where either the diameter of the tracer sphere is large or small compared with 
the mesh size of the network. In the present paper we developed a theory for diffusion of small tracer spheres in open networks of isotropic and nematic networks of rods. It is shown that for such relatively open networks hydrodynamic interactions are dominant over direct interactions (the "shadowing effect"). Furthermore, hydrodynamic interactions are screened due to entanglement of the rods in the network.

Fluorescence correlation spectroscopy (FCS) has been used to probe the long-time self-diffusion coefficient of fluorescently labeled apoferritin in suspensions of fd virus, both in the isotropic and nematic state. A complication for the data analysis is that fd virus by itself fluoresces to some extent. A careful background subtraction is necessary to extract the diffusion coefficient of apoferritin in these mixtures. A cuvette is designed in which a drop of nematic fd-virus suspension is pinned in between two glass plates. It has been a nontrivial step in our experiments to find a procedure to fix a drop of suspension in between two glass plates. A monodomain nematic is prepared by an exposure of the drop to a magnetic field before a FCS measurement.

Combining the FCS data and the theory, the fdconcentration dependence of the hydrodynamic screening length is determined both in the isotropic state and in the nematic state for diffusion parallel and perpendicular to the director. As expected, the screening length decreases with increasing fd concentration in the isotropic state, that is, the penetration depth of a shear wave is less for a denser network. In the nematic state, however, the screening length increases with increasing concentration. This is due to the increase of orientational order as the concentration increases. Apparently, an increasing degree of orientational order strongly decreases hydrodynamic screening. A selfconsistent calculation of the screening length predicts similar features, but is not accurate enough to compare with experiments on a semiquantitative level.

\section{ACKNOWLEDGMENTS}

Financial support from the DAAD and $\mathrm{KBN}$ is gratefully acknowledged. Part of this work is done within the frame work of the European Network of Excellence "Soft Matter Composites" (SoftComp).

\section{APPENDIX A: ON THE ACCURACY OF EQUATION} (17)

There are two approximations involved in Eq. (17): first of all, the $\alpha$ dependence in the bead-index sum in the first term on the right-hand side of Eq. (16) is neglected, and secondly, the second term on the right-hand side in Eq. (16) is neglected against the first term.

The validity of the first approximation is simply verified by calculating the bead-index sums,
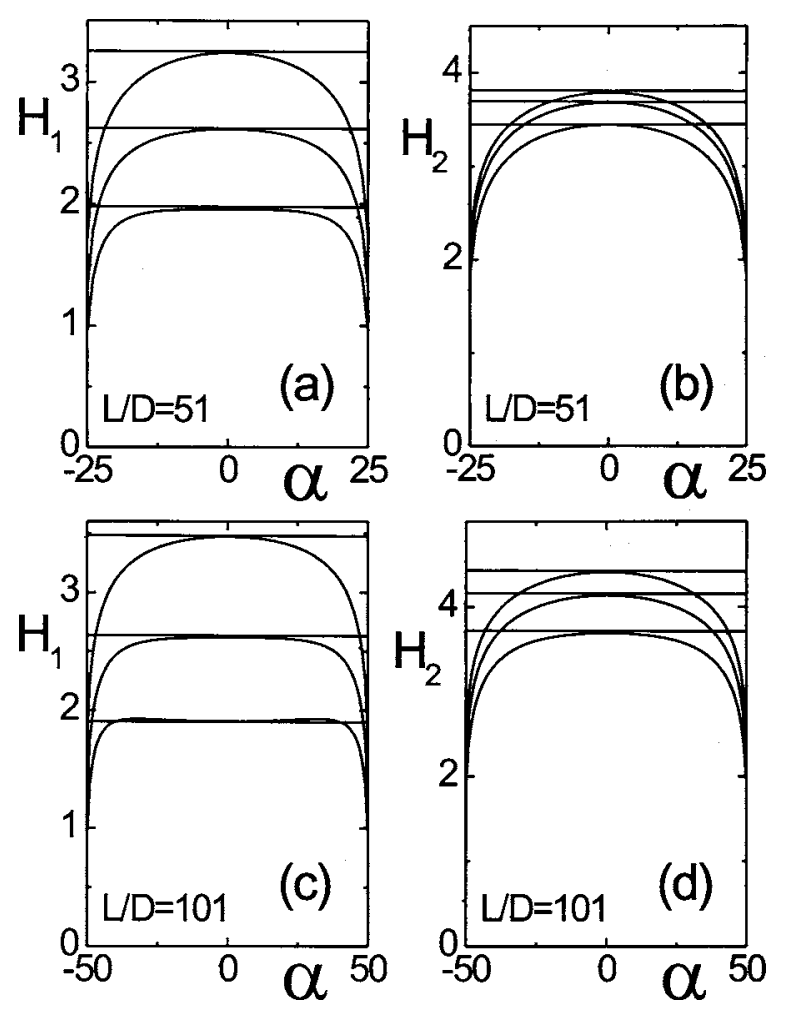

FIG. 11. The $\alpha$ dependence of the sums $H_{1}\left[(\mathrm{a})\right.$ and (c)] and $H_{2}$ [(b) and (d)] in Eq. (A1) for $L / D=51$ [(a) and (b)] and $L / D=101$ [(c) and (d)]. The solid curves in all figures are from bottom to top for $\kappa D=1 / 10,1 / 20$, and $1 / 50$. The horizontal lines indicate the values at $\alpha=0$.

$$
\begin{aligned}
& H_{1}(\alpha) \equiv \sum_{\beta \neq \alpha} \frac{h_{1}(\kappa D|\alpha-\beta|)}{|\alpha-\beta|}, \\
& H_{2}(\alpha) \equiv \sum_{\beta \neq \alpha} \frac{h_{2}(\kappa D|\alpha-\beta|)}{|\alpha-\beta|},
\end{aligned}
$$

as functions of $\alpha$ for various (small) values of $\kappa D$, and compare the numerical values with $H_{1}(\alpha=0)$ and $H_{2}(\alpha=0)$, respectively. The relative difference should be small for the majority of bead-index numbers $\alpha$. As can be seen from Fig. 11, where both $H_{1}$ and $H_{2}$ are plotted as functions of $\alpha$ for $L / D=51$ and 101 , this is indeed the case. In calculating sums with respect to $\alpha$ of functions that do not peak at the edges of the rod, the relative error is of the order of the difference in area under the solid curves and the corresponding straight line that marks the value of $H_{1,2}$ at $\alpha=0$. Sums of $H_{1,2}$ with respect to $\alpha$ differ less than $8 \%$ as compared with the area $(2 m+1) H_{1,2}(\alpha=0)$. Neglecting end effects thus introduces errors of the order of $10 \%$.

Next consider the neglect of the second term in on the right-hand side in Eq. (16) with respect to the first term. Note that it follows from Eq. (22) that, in the absence of hydrodynamic screening, the bead forces $\mathbf{F}_{\beta}^{h}$ typically vary like $\sim 1 /|\mathbf{R} / D-\beta \hat{\mathbf{u}}|$. To estimate the accuracy of the neglect of the second term on the right-hand side of Eq. (16) we shall therefore compare the sum, 

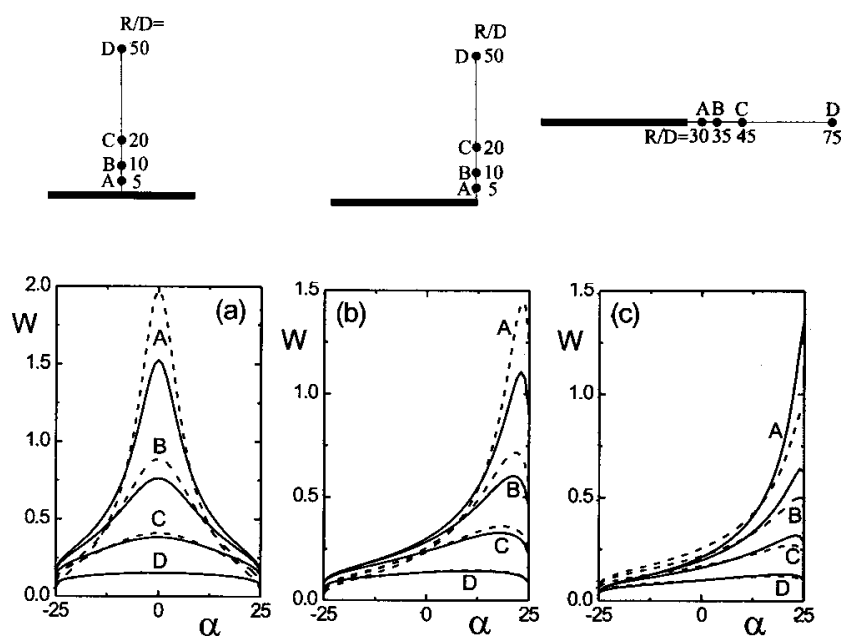

FIG. 12. The integral $W$ in Eq. (A2) where the typical force $\mathbf{F}_{\beta}^{h} \sim 1 / \mid \mathbf{R} / D$ $-\beta \hat{\mathbf{u}} \mid$ is kept in the sum (solid line), and $W$ in Eq. (A3) where the force is taken outside the sum and set equal to $\mathbf{F}_{\alpha}^{h}$ (dashed line). Three different configurations are shown in (a), (b), and (c), as depicted above the graphs, with different distances between the tracer sphere and the rod as indicated by $A, B, C$, and $D$.

$$
W=\sum_{\beta \neq \alpha} \frac{1}{|\mathbf{R} / D-\beta \hat{\mathbf{u}}|} \frac{1}{|\beta-\alpha|},
$$

with its approximation,

$$
W \approx \frac{1}{|\mathbf{R} / D-\alpha \hat{\mathbf{u}}|} \sum_{\beta \neq \alpha} \frac{1}{|\beta-\alpha|},
$$

which is equivalent with the neglect of the second term in Eq. (16). As can be seen from Fig. 12, where $W$ in Eqs. (A2) (solid lines) and (A3) (dashed lines) are plotted for various configurations of the tracer sphere relative to the rod, the relative error in neglecting the second term in Eq. (16) is good to within about $10 \%-20 \%$ for the calculation of diffusion coefficients: the bead-index number dependence is reproduced by the approximate form, and absolute differences are not too large even for close approach of the sphere to the rod. In addition, hydrodynamic screening will improve the accuracy of the approximation, since the "screening functions" $h_{1,2}(\kappa D|\alpha-\beta|)$ in the screened Oseen tensor peak around neighboring beads.

\section{APPENDIX B: DERIVATION OF EQUATION (46)}

Ensemble averages are calculated with respect to the probability function $P\left(\mathbf{r}_{p}\right)=(1 / V) g$, where $V$ is the total volume of the system under consideration. Since the potential $V=0$ for $|z|>\frac{1}{2} L+a$, the ensemble average of the potential force is thus equal to

$$
\begin{aligned}
\langle\nabla V\rangle & =\int d \mathbf{r}_{p} P \nabla V \\
& =\bar{\rho} \int_{-(1 / 2) L-a}^{(1 / 2) L+a} d z \int_{0}^{\infty} d \rho \rho \int_{0}^{2 \pi} d \Psi g(\rho, \Psi) \nabla V(\rho),
\end{aligned}
$$

where as before $\bar{\rho}=N / V$ is the number density of rods. The ensemble average for a single rod is multiplied here by the number of rods $N$ to account for all rods in the system. Substitution of the expression (45) and using that

$$
\nabla=\hat{\boldsymbol{\rho}} \frac{\partial}{\partial \rho}+\hat{\boldsymbol{\Psi}} \frac{1}{\rho} \frac{\partial}{\partial \Psi},
$$

where the unit vectors are equal to $\hat{\boldsymbol{\rho}}=(\cos \{\Psi\}, \sin \{\Psi\}, 0)$ and $\hat{\Psi}=(-\sin \{\Psi\}, \cos \{\Psi\}, 0)$ thus leads to

$$
\begin{aligned}
\langle\nabla V\rangle= & -\beta \mathbf{F}^{\operatorname{ext}} \pi \bar{\rho} L \frac{(a+D / 2)^{1+\nu}}{\nu} \int_{0}^{\infty} d \rho \rho^{1-\nu} \\
& \times \exp \{-\beta V(\rho)\} \frac{d V(\rho)}{d \rho},
\end{aligned}
$$

where it is used that $\mathbf{F}^{\text {ext }}=-\hat{\mathbf{e}}_{1} F^{\text {ext }}$ [see Fig. 4(c)]. Here, $L$ $+2 a$ is set equal to $L$, since $2 a / L \ll 1$. For hard-core interactions we have (with $\delta$ the delta distribution)

$$
\begin{aligned}
\exp \{-\beta V(\rho)\} \frac{d V(\rho)}{d \rho} & =-\beta^{-1} \frac{d \exp \{-\beta V(\rho)\}}{d \rho} \\
& =-\beta^{-1} \delta(\rho-a-D / 2) .
\end{aligned}
$$

Substitution into Eq. (B3) thus gives

$$
\beta\langle\nabla V\rangle=\beta \mathbf{F}^{\mathrm{ext}} \pi \bar{\rho} L \frac{(a+D / 2)^{2}}{\nu} .
$$

Expressing the number concentration $\bar{\rho}$ in terms of the volume fraction $\varphi=(\pi / 4) D^{2} L \bar{\rho}$ of rods leads to the first of the two expressions in Eq. (46).

Similarly to Eq. (B1) the ensemble average of the Brownian force is equal to

$$
\begin{aligned}
\langle\nabla \ln \{g\}\rangle & =\int d \mathbf{r}_{p} P \nabla \ln \{g\} \\
& =\bar{\rho} \int_{-(1 / 2) L-a}^{(1 / 2) L+a} d z \int_{0}^{\infty} d \rho \rho \int_{0}^{2 \pi} d \Psi \nabla g(\rho, \Psi) .
\end{aligned}
$$

Substitution of Eq. (45) for the pair-correlation function, transforming the differentiation to cylindrical coordinates [see Eq. (B2)], and using that for hard-core interactions,

$$
\frac{d \exp \{-\beta V(\rho)\}}{d \rho}=\delta(\rho-a-D / 2),
$$

it is readily found by a single partial integration that there is a cancellation of terms in Eq. (B6), leading to a zeroensemble-averaged Brownian force, that is, $\langle\nabla \ln \{g\}\rangle=0$.

\section{APPENDIX C: A SELF-CONSISTENT CALCULATION OF THE SCREENING LENGTH}

In this Appendix we shall calculate the friction coefficient $\lambda$ in Eq. (3), which then identifies the hydrodynamic screening length $\kappa^{-1}$ through Eq. (5). Since the network of the long and thin rods is very open, hydrodynamic interactions through fields that are multiply reflected by different rods towards the sphere are neglected. The friction coefficient $\lambda$ can thus be approximated as the friction coefficient of a single rod in a Debye-Büche-Brinkman fluid. 
Neglecting end effects, the forces $\mathbf{F}_{\alpha}^{h}$ of bead number $\alpha$ on the fluid in an otherwise uniform fluid flow is equal to $\mathbf{F}(D / L)$ for each bead, where $\mathbf{F}$ is the total force that a single rod exerts on the fluid. Since the fluid flow $\mathbf{u}_{0}$ in Eq. (15) is now a constant $\mathbf{u}$, this "integral equation" reduces to

$$
\frac{\mathbf{F}}{3 \pi \eta_{0} L}+\mathbf{u}+\frac{D}{L} \sum_{\beta \neq \alpha} \mathbf{T}_{s}(D \hat{\mathbf{u}}(\alpha-\beta)) \cdot \mathbf{F}=0 .
$$

As in the previous subsection, end effects can be neglected for the long and thin rods under consideration and the beadindex sum can be rewritten as an integral. Analogous to Eq. (22) it is found that (for convenience the screening length dependence of $G$ and $H$ are not denoted here explicitly)

$$
\mathbf{F}=-\frac{2 \pi \eta_{0} L}{G}\left[\hat{\mathbf{I}}-\frac{H}{G+H} \hat{\mathbf{u}} \hat{\mathbf{u}}\right] \cdot \mathbf{u},
$$

where $G$ and $H$ are defined in Eq. (20). Orientational averaging with respect to $\hat{\mathbf{u}}$ identifies the effective friction coefficient through Eq. (3) and thereby the hydrodynamic screening through Eq. (5). Introducing the tensor

$$
\mathbf{Q}=\frac{3}{2}\left[\langle\hat{\mathbf{u}} \hat{\mathbf{u}}\rangle-\frac{1}{3} \hat{\mathbf{I}}\right],
$$

orientational averaging of Eq. (C2) for a given velocity $\mathbf{u}$ leads to [with, for example, $\left.G_{\|}=G\left(\kappa_{\|} D, \kappa_{\|} L\right)\right]$,

$$
\begin{aligned}
& \lambda_{\text {iso }}=2 \pi \eta_{0} L \frac{G_{\text {iso }}+(2 / 3) H_{\text {iso }}}{G_{\text {iso }}\left[G_{\text {iso }}+H_{\text {iso }}\right]}, \\
& \lambda_{\|}=2 \pi \eta_{0} L \frac{G_{\|}+(2 / 3) H_{\|}\{1+(1 / 2) S\}}{G_{\|}\left[G_{\|}+H_{\|}\right]}, \\
& \lambda_{\perp}=2 \pi \eta_{0} L \frac{G_{\perp}+(2 / 3) H_{\perp}\{1-S\}}{G_{\perp}\left[G_{\perp}+H_{\perp}\right]},
\end{aligned}
$$

where the subscripts "iso," "\|l" and " $\perp$ " refer to the isotropic state and the nematic state with diffusion parallel and perpendicular to the nematic director, respectively. Here, it is assumed that for diffusion in the nematic state, the most important contributions originate from scattering where $\mathbf{F}$ is colinear to the velocity field $\mathbf{u}$. Furthermore, $S$ is the largest eigenvalue of $\mathbf{Q}$, the "scalar orientational order parameter," which measures the degree of orientational order. Equation (5) for the screening length in terms of the friction coefficient thus leads to the following self-consistent relations,

$$
\begin{aligned}
& \left(\kappa_{\text {iso }} L\right)^{2}=12 \frac{\bar{\rho}}{\bar{\rho}^{\star}} \frac{G_{\text {iso }}+(2 / 3) H_{\text {iso }}}{G_{\text {iso }}\left[G_{\text {iso }}+H_{\text {iso }}\right]}, \\
& \left(\kappa_{\|} L\right)^{2}=12 \frac{\bar{\rho}}{\bar{\rho}^{\star}} \frac{G_{\|}+(2 / 3) H_{\|}\{1+(1 / 2) S\}}{G_{\|}\left[G_{\|}+H_{\|}\right]}, \\
& \left(\kappa_{\perp} L\right)^{2}=12 \frac{\bar{\rho}}{\bar{\rho}^{\star}} \frac{G_{\perp}+(2 / 3) H_{\perp}\{1-S\}}{G_{\perp}\left[G_{\perp}+H_{\perp}\right]},
\end{aligned}
$$

where the index on $\kappa$ again refers to the type of diffusion and where

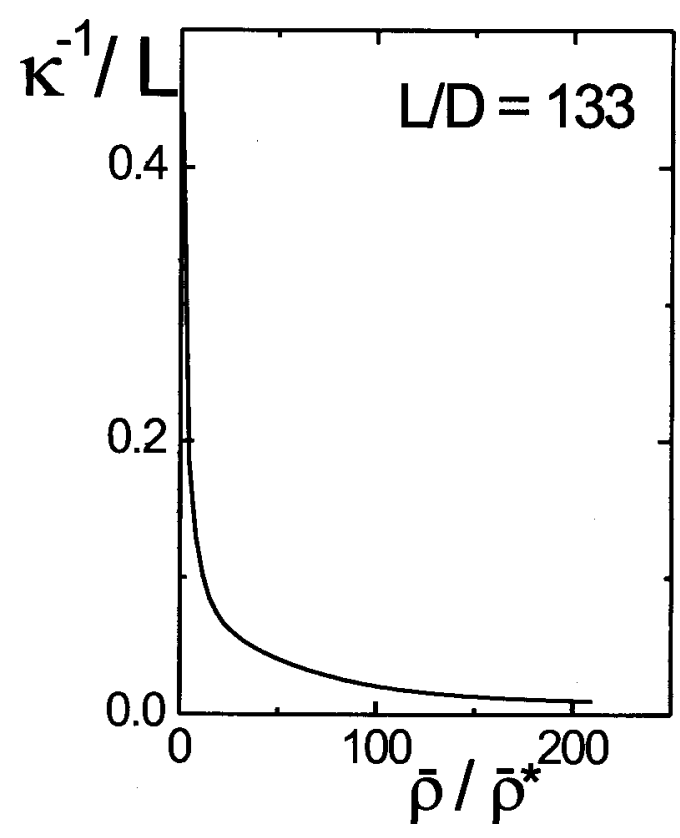

FIG. 13. The hydrodynamic screening length $\kappa^{-1}$ in units of the length $L$ of the rods as a function of the number concentration $\bar{\rho}$ in units of the overlap concentration $\bar{\rho}^{\star}$. The parameters comply with a fd-virus network, where $L / D=133$ and $\bar{\rho}^{\star}=0.076 \mathrm{mg} / \mathrm{ml}$. The lower isotropic-nematic binodal concentration is equal to $\bar{\rho} / \bar{\rho}^{\star}=263$.

$$
\bar{\rho}^{\star}=6 /\left(\pi L^{3}\right)
$$

is the overlap concentration. For fd-virus suspensions the overlap concentration is $0.076 \mathrm{mg} / \mathrm{ml}$. The binodals for the isotropic-nematic phase transition are located at 20 and $22 \mathrm{mg} / \mathrm{ml}$, corresponding to $\bar{\rho} / \bar{\rho}^{\star}=263$ and 303 , respectively.

The self-consistency relations (C5) must be solved numerically. As can be seen from Fig. 13 and the experimental Fig. 10, the numerical values of the self-consistently calculated screening length agrees with the experiments only for very low concentration of fd virus. For higher concentrations in the isotropic state, the screening length becomes of the order of the diameter of fd virus, where the screening concept obviously breaks down. Furthermore, the screening lengths in the nematic state for diffusion perpendicular to the director are similarly of the order of the thickness of the rod, while for diffusion parallel to the director Eq. (C5) has no solutions. It thus seems that a self-consistent calculation of the screening length does not work for the open network under consideration. The probable reason for this is that the hydrodynamic field that a rod experiences is never homogeneous over its whole length. A simple calculation of the friction coefficient of a rod in an otherwise quiescent (screened) fluid is therefore unrealistic.

${ }^{1}$ C. F. Schmidt, M. Bärmann, G. Isenberg, and E. Sackmann, Macromolecules 22, 3638 (1989).

${ }^{2}$ J. D. Jones and K. Luby-Phelps, Biophys. J. 71, 2742 (1996).

${ }^{3}$ O. Seksek, J. Biwersi, and A. S. Verkman, J. Cell Biol. 138, 131 (1997).

${ }^{4}$ F. G. Schmidt, B. Hinner, and E. Sackmann, Phys. Rev. E 61, 5646 (2000).

${ }^{5}$ J. L. McGrath, J. H. Hartwig, and S. C. Kuo, Biophys. J. 79, 3258 (2000).

${ }^{6}$ Y. Shafrir, D. ben-Avraham, and G. Forgacs, J. Cell. Sci. 113, 2747 (2000). 
${ }^{7}$ M. Arrio-Dupont, G. Foucault, M. Vacher, P. F. Deveaux, and S. Cribier, Biophys. J. 78, 901 (2000).

${ }^{8}$ J. Apgar, Y. Tseng, E. Fedorov, M. B. Herwig, S. C. Almo, and D. Wirtz, Biophys. J. 79, 1095 (2000).

${ }^{9}$ Y. Tseng and D. Wirtz, Biophys. J. 81, 1643 (2001).

${ }^{10}$ A. S. Verkman, Trends Biochem. Sci. 27, 27 (2002).

${ }^{11}$ I. Y. Wong, M. L. Gardel, D. R. Reichman, E. R. Weeks, M. T. Valentine, A. R. Bausch, and D. A. Weitz, Phys. Rev. Lett. 92, 178101-1 (2004).

${ }^{12}$ Z. Dogic, Ph.D. thesis, Brandeis University, 2000.

${ }^{13}$ J. Tang and S. Fraden, Liq. Cryst. 19, 459 (1995).

${ }^{14}$ M. Adams, Z. Dogic, S. L. Keller, and S. Fraden, Nature (London) 393 349 (1998).

${ }^{15}$ Z. Dogic and S. Fraden, Langmuir 16, 7820 (2000).

${ }^{16}$ Z. Dogic, K. R. Purdy, E. Grelet, M. Adams, and S. Fraden, Phys. Rev. E 69, 051702 (2004).

${ }^{17}$ C. Graf, H. Kramer, M. Deggelmann, M. Hagenbüchle, C. Johner, C. Martin, and R. Weber, J. Chem. Phys. 98, 4920 (1993).

${ }^{18}$ F. G. Schmidt, B. Hinner, E. Sackmann, and J. X. Tang, Phys. Rev. E 62, 5509 (2000)

${ }^{19}$ T. A. J. Lenstra, Z. Dogic, and J. K. G. Dhont, J. Chem. Phys. 114, 10151 (2001)

${ }^{20}$ K.-H. Lin, J. C. Crocker, A. C. Zeri, and A. G. Yodh, Phys. Rev. Lett. 87, 088301-1 (2001).

${ }^{21}$ A. R. Altenberger and M. Tirrell, J. Chem. Phys. 80, 2208 (1984).

${ }^{22}$ S. Kim and W. B. Russel, J. Fluid Mech. 154, 269 (1985).

${ }^{23}$ A. R. Altenberger, M. Tirrell, and J. S. Dahler, J. Chem. Phys. 84, 5122 (1986).

${ }^{24}$ R. Mauri, J. Eng. Math. 33, 103 (1996).

${ }^{25}$ A. G. Ogston, B. N. Preston, and J. D. Wells, Proc. R. Soc. London, Ser A 333, 297 (1973).

${ }^{26}$ R. I. Cukier, Macromolecules 17, 252 (1984).

${ }^{27}$ J. Han and J. Herzfeld, Biophys. J. 65, 1155 (1993)

${ }^{28}$ K. K. S. Buck, S. R. Dungan, and R. J. Phillips, J. Fluid Mech. 396, 287 (1999) [see their Eq. 28: in the gradient contribution there is a factor $a^{2}$ missing, that is, the prefactor $1 / 6$ should be $a^{2} / 6$ ]

${ }^{29}$ J. G. Kirkwood and J. Riseman, J. Chem. Phys. 16, 565 (1948).

${ }^{30}$ B. U. Felderhof and J. M. Deutsch, J. Chem. Phys. 62, 2391 (1975).

${ }^{31}$ K. Kang, J. Gapinski, M. P. Lettinga, J. Buitenhuis, G. Meier, M. Ratajczyk, J. K. G. Dhont, and A. Patkowski, J. Chem. Phys. 122, 044905-1 (2005).

${ }^{32}$ S. Gorti and B. R. Ware, J. Chem. Phys. 83, 6449 (1985).

${ }^{33}$ T. F. Kosar and R. J. Phillips, AIChE J. 41, 701 (1994).

${ }^{34}$ B. Schnurr, F. Gittes, F. C. MacKintosch, and C. F. Schmidt, Macromolecules 30, 7781 (1997)

${ }^{35}$ E. M. Johnson, D. A. Berk, R. K. Jain, and W. M. Deen, Biophys. J. 70,
1017 (1996).

${ }^{36}$ A. Gennerich and D. Schild, Biophys. J. 83, 510 (2002).

${ }^{37}$ J. van der Gucht, N. A. M. Besseling, W. Knoben, L. Bouteiller, and M. A. Cohen Stuart, Phys. Rev. E 67, 051106 (2003).

${ }^{38}$ S. Mangenot, S. Keller, and J. Rädler, Biophys. J. 85, 1817 (2003).

${ }^{39}$ G. H. Koenderink, S. Sacanna, D. G. A. Aarts, and A. P. Philipse, Phys. Rev. E 69, 021804 (2004).

${ }^{40}$ M. P. B. van Bruggen, H. N. W. Lekkerkerker, and J. K. G. Dhont, Phys. Rev. E 56, 4394 (1997).

${ }^{41}$ M. P. B. van Bruggen, H. N. W. Lekkerkerker, G. Maret, and J. K. G. Dhont, Phys. Rev. E 58, 7668 (1998).

${ }^{42}$ C. Lellig, J. Wagner, R. Hempelmann, S. Keller, D. Lumma, and W. Haërtl, J. Chem. Phys. 121, 7022 (2004).

${ }^{43}$ M. P. Lettinga, E. Barry, and Z. Dogic, Europhys. Lett. 71, 692 (2005).

${ }^{44}$ G. K. Batchelor, J. Fluid Mech. 52, 245 (1972); 74, 1 (1976); 131, 155 (1983). A corrigendum to the last paper can be found in J. Fluid Mech. 137, 467 (1983).

${ }^{45}$ T. Odijk, Macromolecules 19, 2313 (1986).

${ }^{46}$ L. Onsager, Ann. N.Y. Acad. Sci. 51, 627 (1949); Phys. Rev. 62, 558 (1942).

${ }^{47}$ K. R. Purdy, Z. Dogic, S. Fraden, A. Rühm, L. Lurio, and S. G. J. Mochrie, Phys. Rev. E 67, 031708 (2003).

${ }^{48}$ K. Zimmermann, J. Hagedorn, C. C. Heuck, M. Hinrichsen, and J. Ludwig, J. Biol. Chem. 261, 1653 (1986).

${ }^{49}$ J. Sambrook, E. F. Fritsch, and T. Maniatis, Molecular Cloning: A Laboratory Manual (Cold Spring Harbor Laboratory, New York, 1989).

${ }^{50}$ C. de Haen, Anal. Biochem. 166, 235 (1987).

${ }^{51}$ S. H. Kilcoyne, G. R. Mitchell, and R. Cywinski, Physica B 180/181, 767 (1992).

${ }^{52}$ T. Takahashi, M. Hogyoku, and K. Nagayama, J. Cryst. Growth 168, 130 (1996).

${ }^{53}$ P. D. Hempstead, S. J. Yewdall, A. R. Fernie, D. M. Lawson, P. J. Artymiuk, D. W. Rice, G. C. Ford, and P. Harrison, J. Mol. Biol. 288, 424 (1997).

${ }^{54}$ D. N. Petsev, B. R. Thomas, S.-T. Yau, and P. G. Velikov, Biophys. J. 78, 2060 (2000)

${ }^{55}$ W. Häussler, A. Wilk, J. Gapinski, and A. Patkowski, J. Chem. Phys. 117, 413 (2002).

${ }^{56}$ Since the mesh size scales with $1 / \sqrt{c}$ [see P.-G. de Gennes, P. Pincus, and R. M. Velasco, J. Phys. (France) 37, 1461 (1976)], one can write $\xi$ $=K / \sqrt{c}$. Just above the overlap concentration, $\xi \approx L$, so that $K \approx L \sqrt{c^{\star}}$.

${ }^{57}$ M. P. Lettinga, Z. Dogic, H. Wang, and J. Vermant, Langmuir 21, 8048 (2005) [see their Fig. 6a].

${ }^{58}$ J. K. G. Dhont and W. J. Briels, Colloids Surf., A 213, 131 (2003). 